\title{
Organisationen, Personen und Biographien: Entwicklungsvarianten von Inklusionsverhältnissen ${ }^{1}$
}

\author{
Hanns-Georg Brose, Ursula Holtgrewe, Gabriele Wagner
}

Gerhard-Mercator-Universität - Gesamthochschule Duisburg, FB 1, Fach: Soziologie, Lotharstr. 65, D-47048 Duisburg

Zusammenfassung: Der Beitrag beleuchtet das Verhältnis von Organisation und Biographie unter dem Gesichtspunkt der Inklusion. Nimmt die Organisationstheorie herkömmlicherweise an, daß gerade die Exklusion von Persönlichem, Subjektivem und „Privatem“ moderne Organisationen kennzeichnet, so untersuchen wir anhand von vier Beispielen die vielfältigen Weisen der Kopplung der Arbeit in Organisationen mit der biographischen Entwicklung und außerbetrieblichen Lebensführung von Personen. Während die Beamten des 19. Jahrhunderts und die Mitglieder japanischer „Betriebsclans“ total inkludiert, also in ihrer Gesamtheit langfristig von der Organisation in Anspruch genommen werden, werden weibliche Beamte und Zeitarbeiter nur partial oder tangential an die Organisationen angeschlossen. Inklusionsverhältnisse werden auf der Seite der Organisationen durch Rekrutierungs- und Sozialisationsprozesse, Belohnungen und Versprechungen charakterisiert, auf der Seite der Mitglieder durch subjektive Dispositionen und biographische Skripte. Es ergeben sich jeweils spezifische Wechselwirkungen und Tauschbeziehungen.

\section{Einleitung}

Es fällt nicht schwer, die von uns behandelte Fragestellung als Variation eines "klassischen" und lange Zeit prominenten Themas zu erkennen: des Themas „Individuum und Organisation“ (Adorno 1953; Argyris 1957; 1973; Schimank 1981). Unser Beitrag bemüht sich freilich darum, dieses Verhältnis, das häufig als Konflikt zwischen Organisationsstrukturen einerseits und menschlichen Ausstattungen und Bedürfnissen andererseits diskutiert wird, aus einem spezifischen Blickwinkel zu betrachten.

Es geht uns zum einen darum, (historisch) verschiedene Entwicklungsvarianten dieser Beziehung darzustellen und damit ihre Veränderlichkeit $\mathrm{zu}$ betonen. Zum anderen soll hervorgehoben werden, daß die Inklusion von Personen in Organisationen nicht einmal und für allemal begründet wird. Vielmehr ist davon auszugehen, daß sich Organisationen und Personen auf unterschiedliche Weise entwickeln können. Wir halten es daher für aufschlußreich, die Unterschiedlichkeit der Lebenszyklen von Organisationen und Personen und die Form ihrer Synchronisation zu untersuchen.

1 Es handelt sich um die überarbeitete Fassung eines Vortrags, den die Autoren auf der Jahrestagung der Sektion Biographieforschung der DGS „Biographien in Institutionen" im September 1993 in Mainz gehalten haben. Wir danken Christoph Deutschmann, Regine Mathias, Monika Wohlrab-Sahr und den anonymen Gutachtern der ZfS für ihre kritischen und hilfreichen Anmerkungen. „Fehler und andere Ungenauigkeiten“ haben selbstverständlich allein wir zu verantworten.
Am Anfang steht die Fragestellung nach dem Inklusionsverhältnis von Gesellschaft und Individuum bzw. nach dem Verhältnis von Arbeit - als dem an der Schwelle zur Moderne zentralen gesellschaftlichen Inklusionsmechanismus - und Lebensführung. Diese, einstmals ständisch geregelte, Lebensführung wird erst durch die Ausdifferenzierung gesellschaftlicher Funktionssysteme und formaler Organisationssysteme zum Problem.

Sowohl in segmentär als auch in stratifikatorisch differenzierten Gesellschaften gehört die Einzelperson einem einzigen gesellschaftlichen Bereich an. „Die Individualität ist mit Namen, Bekanntsein, Rechten und Pflichten und vor allem mit Aufgehobensein in einem Kontext von Leistungen und Gegenleistungen durch soziale Inklusion gegeben“ (Luhmann 1989: 156). In funktional differenzierten Gesellschaften hingegen haben die Personen gleichzeitig an verschiedenen Systemen teil: Als Rechtssubjekte sind sie in das Rechtssystem, als Wähler sind sie in das politische System und als Produzenten/Konsumenten sind sie in das Wirtschaftssystem inkludiert (Stichweh 1988). Im Kontext dieser Gesellschaftsstruktur fällt ihnen die Aufgabe zu, die Teilhabe an den ausdifferenzierten Funktionssystemen - mit deren eigenen Logiken und Semantiken - aufeinander zu beziehen und zu ordnen. Diese Gestaltungsleistung ist Kern der Lebensführung. In ihrer Systematisierung erblickte Weber ja die lebensweltlich und kulturell notwendige Ergänzung des ökonomischen und organisatorischen Rationalisierungsprozesses. Damit kommt auch die Biographie ins Spiel. Denn wenn die Inklusion in die Gesellschaft durch den 
„Geburtsstand nicht mehr gegeben ist" (Luhmann 1989: 190), wird es sozial relevant, über Herkunft und Zukunft von Personen, über lebensgeschichtliche Erfahrungen und über Erwartungen im Horizont der Lebenszeit nachzudenken. Dies läßt sich in der gesellschaftlichen Semantik, in den Literaturgattungen des Entwicklungsromans und der Biographik nachzeichnen. Biographie reflektiert dann den Lebenslauf vor dem Problemhorizont kontingenter Formen der Lebensführung und wird zu ihrem „,notwendigen “ Bestandteil. ${ }^{2}$

Mit der funktionalen Differenzierung geht die Systembildung auf der Ebene von Organisationen einher (Luhmann 1981), und diese Ausdifferenzierung formaler Organisationen wird ihrerseits zu einer dominanten Entwicklungslinie (Perrow 1991). Die Inklusion in die Funktionssysteme, insbesondere in das Wirtschaftssystem, erfolgt - wegen der Geldabhängigkeit der Wirtschaftssubjekte - fast nur noch über die Mitgliedschaft in Organisationen. Die Inklusion in die Gesellschaft und deren Funktionssysteme wird also über die Mitgliedschaft in Organisationen spezifiziert (Inklusion qua Exklusion) und wirkt selektiv. Wie sich diese Eng-Führung des Lebens durch die Mitgliedschaft in (Arbeits-)Organisationen auswirkt, und wieviel an Lebenszusammenhängen gekappt oder doch gekoppelt wird, darum soll es in unserem Beitrag gehen.

Um einen Ausgangspunkt ins Auge zu fassen, werfen wir den Blick zunächst kurz zurück und betrachten das Zunftwesen unter dem Gesichtspunkt, inwieweit an den Zünften bereits Elemente formaler Organisation erkennbar sind (vgl. Kieser

2 Der Begriff der Lebensführung bietet den Vorteil, biographietheoretische Überlegungen in Kontakt mit der klassischen Modernisierungstheorie Webers zu bringen. Aus diesem Grund wird er hier aufgegriffen. In der Diskussion der letzten Jahre hat Voß (1991) diesen Begriff stark gemacht, ihn aber unverständlicherweise immer wieder - in ausdrücklicher Abgrenzung zu diachronen Betrachtungen - auf die „alltägliche“ Lebensführung eingeengt. Natürlich muß die Verknüpfung von Lebensbereichen immer auch im Alltag vollzogen werden. Aber wie dabei die Relevanzen strukturiert sind, erschließt sich nicht ohne den Bezug zur Lebenszeit. Wenn Weber von alltäglicher Lebensführung spricht, dann grenzt er diese gegen die Außeralltäg. lichkeit virtuoser bzw. religiöser Lebensführung $a b$, bezieht sich also primär auf unterschiedliche Wertsphären und nicht auf die zeitliche Struktur des Alltags. Dagegen verweist der Aspekt der Systematisierung der alltäglichen Lebensführung - als säkularisierte Orientierung an der Jenseitigkeit - gerade über die zeitliche Struktur des Alltags hinaus.
1989), und wie sich die Zugehörigkeit zur Zunft auf die Lebensführung ihrer Mitglieder auswirkte.

Zünften fehlt das Merkmal der Freiwilligkeit der Mitgliedschaft; zugeschriebene Merkmale der Herkunft sind Bedingung für die Mitgliedschaft, die durch Initiation und nicht durch Schließung eines Vertrags begründet wird. Qualifikationen werden durch Erfahrungs- und Imitationslernen und nicht in systematischer Ausbildung erworben. Die Reproduktion der einzelnen Haushalte/Betriebe wird durch Zwang zur Mobilität (Gesellenwanderung) und Begrenzung der Niederlassungs- und Heiratschancen, also durch kollektiv verbindliche Formung der Lebensweise von Personen, gesteuert. Riten und symbolische Praktiken tragen zur Stabilisierung dieser Lebensführung bei. Damit wollen wir andeuten, daß die zünftige Lebensweise - übrigens bis weit über die Schwelle des Industriezeitalters hinaus - eine enge Kombination von $A r$ beit und Lebensführung bestehen ließ.

Die weitere Entwicklung hat eine solche Einheit dann mehr und mehr gesprengt. Das ist unter dem Stichwort der „Freisetzung“ und der Entwicklung zur vertragsförmig geregelten, „freien Lohnarbeit" von Marx und Weber analysiert worden und auch in Luhmanns systemtheoretischer Organisationsanalyse Bezugspunkt geblieben (vgl. Gabriel 1979). Die Merkmale dieser dominanten Entwicklungslinie im Verhältnis von Personen und Organisationen sind:

- Die Trennung von Amt/Aufgabe und Person;

- Die Reinigung der sachlichen Arbeit von allen persönlichen, irrationalen und emotionalen Elementen;

- Definition von Verhaltenserwartungen in der Form von Arbeitsrollen und deren personenunabhängige Generalisierung als Stellen;

- Zweckindifferente Motivierung der Mitgliederbeiträge: ${ }^{3}$

- Koordination der Mitgliederbeiträge in einer hierarchisch geordneten Kommunikations- bzw. Anweisungsstruktur;

- Entkopplung von innerbetrieblicher Führung und außerbetrieblicher Lebensweise bzw. die Trennung von Arbeitszeit und Freizeit.

Im Zuge der Entwicklung formaler Organisationen vollzieht sich die Exklusion des Persönlichen,

3 Ein Punkt, den besonders Luhmann (1973) hervorhebt. Gerade hier wird die Differenz zwischen Organisationen und Institutionen (Perrow 1979: $186 \mathrm{ff}$ ) bzw. Organisationen und freiwilligen Vereinigungen gesehen (Knoke/Prensky 1984). 
des Irrationalen und wird in der Theorie der Rationalisierung und Bürokratisierung als Fortschritt gepriesen. In den Arbeitswelten der Industrialisierung kehrt das Ausgeschlossene jedoch als bedrohlicher Opportunismus, als Bremsen und shirking wieder. Ist das Treueverhältnis und Vertrauen dahin, wird die Kontrolle nötig. Hier sind die Konfliktlinien zwischen Personen und Organisationen gezogen und hier findet statt, was als die Domestizierung der Individuen durch die Organisationen kritisiert und Entfremdung genannt wird.

Vertragstheoretisch läßt sich dies als Kontrollproblem interpretieren, das durch die Unbestimmtheit des Arbeitsvertrags hervorgerufen wird. Von daher wird auch die Verbreitung und Effizienz der Arten von Belohnung plausibilisiert, die die Erwartungen und Entscheidungen der Organisationsmitglieder durch längerfristig wirkende Anreize zu steuern versuchen: Senioritätslöhne, Karrieremechanismen etc. Die Disziplinierung erfolgt an der langen Leine der deferred gratification pattern und sie wird von außen nach innen verlagert. Entstanden ist diese Verlagerung wohl nicht primär aus einem „Paradigmawechsel“ der betrieblichen Personalführung, sondern aus Mischformen und der Überlagerung unterschiedlicher und ungleichzeitiger Steuerungsmechanismen über den Lohnanreiz hinaus.

So entstehen die Verhaltens- und Dienstvorschriften der Angestellten (auch) aus der Tradition der frühen Industriebeamten, und diese wiederum konnten durch Modelle militärischer Hierarchie und feudaler Verpflichtungen inspiriert sein (Kokka 1969). Selbst für die lohnanreizstimulierten Fabrikarbeiter tayloristischer Prägung galt, daß deren außerbetriebliche Lebensführung $-z$.B. der Alkoholkonsum - durch die fordistischen $\mathrm{Be}$ triebsingenieure kontrolliert wurde (Siegel 1993). Insgesamt baut ja das Gesellschaftsmodell des Fordismus auf einer positiven Rückkopplung von Produktion und Konsumtion auf. Arbeit und Lebensführung verweisen hier aufeinander (vgl. u.a. Sachse 1988). Auch die paternalistischen, auf statusförmiger Inklusion beruhenden Beschäftigungsverhältnisse, wie sie am Beispiel betrieblicher Sozialpolitiken - mit ihren Werkswohnungen und Betriebsrenten - belegt worden sind, machen deutlich, daß die Wirklichkeit der industriellen Arbeitswelt eine große Vielfalt und Vielzahl von Beschäftigungsverhältnissen hervorbrachte, die weit von der Lohnarbeit sans phrase entfernt sind. ${ }^{4}$

4 Ein neueres Beispiel geben Balazs/Faguer (1991).
Dieser Strang der Entwicklung und die ihn interpretierende soziologische Argumentation ist bekannt. Wir wollen uns hier verschiedenen Seitenlinien der Entwicklung zuwenden. Dabei geht es zunächst um Beispiele, in denen diese Entwicklung sich nicht voll durchgesetzt hat, und eine mehr oder weniger enge Kopplung von Arbeit in Organisationen mit der biographischen Entwicklung von Personen bzw. ihrer Lebensführung zu erkennen ist.

Dafür steht das (historische) Beispiel der Beamten, mit dem wir beginnen (2.). Am Beispiel der Reichspost wollen wir dann im folgenden skizzieren, wie eine Organisation auf Veränderungen in ihrer technischen Ausstattung durch Inklusion eines besonderen Typs von Mitgliedern, nämlich weiblichen Beamten, zu reagieren versucht, wie dabei einerseits Lemprozesse der Organisation in Gang gesetzt werden, und andererseits Chancen der Individualisierung weiblicher Lebensführung entstehen können (3.). Solche Formen der - mehr oder weniger engen - Beziehungen und Wechselwirkungen von Organisationsentwicklung und biographischer Entwicklung werden wir am Schluß in einer aktuellen Variante, am Beispiel des Clanmodells - wieder aufgreifen (5.). Damit wollen wir die Möglichkeit andeuten, daß die im Modernisierungsproze $\beta$ aus den Grenzen der Organisation ausgeschlossene Biographie wieder in diese eintritt, mit ihr in einer Form der Totalinklusion nachgerade verschmilzt.

Das - vorher skizzierte - Beispiel der Zeitarbeit soll dagegen zeigen (4.), daß das, was nach herrschender Interpretation eigentlich immer schon geschieht, nämlich die völlige Entkopplung von Organisation und Lebensführung, ein spezielles Arrangement vorauszusetzen scheint, also keineswegs selbstverständliches Resultat der Modernisierung ist. Und selbst in dieser Variante, der Zeitarbeit, ist die Indifferenz von Biographie und Organisation nicht die ganze Wahrheit. Auch in dieser Form der Beschäftigung scheinen sich wiederum spezifische Synergieeffekte von biographischer und organisationaler Entwicklung ergeben zu können.

Folgende Dimensionen von Inklusionsverhältnissen sind im Vorgriff auf Abschnitt 6. zu nennen: Organisationen und Biographien werden zunächst über Rekrutierung und interne Sozialisation, Entlohnung, Karriere und soziale Absicherung verkoppelt. Über diese „materiellen“ Aspekte hinaus werden Organisationsmitglieder auch über symbolische Ausstattungen des Beschäftigungsverhält- 
nisses und historisch spezifische Relationen sozialen Tausches inkludiert. Es ergeben sich jeweils charakteristische Wechselwirkungen zwischen Organisation und Biographie, aus denen überindividuelle biographische Skripte entstehen. Solche biographischen Skripte müssen und können immer noch von den Subjekten umgesetzt werden. In unserem Beitrag steht jedoch nicht diese Seite subjektiver Biographisierungsleistungen, sondern die der organisationellen Vorgaben im Vordergrund.

Zwischen den von uns aufgespannten Polen der Tangentialinklusion (in der Zeitarbeit) und der Totalinklusion (im Clan), ist also ein Kontinuum von Inklusionsverhältnissen anzusiedeln. Seine Dimensionen im Wege der Minimal- und Maximalkontrastierung zu erschließen, war Ziel unserer - kontingenten, aber nicht willkürlichen - Fallauswahl. Diese Fälle sind zwar typisierend zugespitzt, doch ist der Leser aufgefordert, ein Stück weit der Eigenlogik unserer Darstellungen zu folgen. Aufgrund ihrer historischen und sozialen Unterschiedlichkeit wollen wir die ausgewählten Inklusionsverhältnisse - entsprechend dem induktiven Gang unserer eigenen Analyse - nicht von vornherein in einem allzu rigiden Raster präsentieren.

\section{Beamte}

Die Beamtenschaft ist - insbesondere seit dem 18. und 19. Jahrhundert - mit der Herausbildung formaler Organisationen in Westeuropa verbunden. In den Verwaltungen der nunmehr entstandenen Territorialstaaten bedeutet Rationalisierung die Herausbildung des Weberschen Idealtyps von Bürokratie und der dazu passenden Organisationsmitglieder. Dies geschieht nun nicht in der Form der Freisetzung von ständischen, familiären usw. Bindungen wie beim „freien Lohnarbeiter“. Vielmehr werden vormoderne Elemente der Inklusion wie Treue, Fürsorge, ständisches Recht mit der Ausdehnung der modernen Bürokratie aktualisiert und auf neue Bedienstetengruppen ausgedehnt, so daß wir bei den Beamten von einer Rationalisierung ohne Freisetzung sprechen können. ${ }^{5}$

Idealtypisch werden Beamte nicht auf freien Arbeitsmärkten rekrutiert und per Vertrag für bestimmte Tätigkeiten eingestellt, sondern per Hoheitsakt auf Lebenszeit in den Staatsdienst ver-

5 Die Darstellung folgt, wo nicht anders angegeben, Weber 1972; Wunder $1974 ; 1977 ; 1986$ und Hattenhauer 1980. pflichtet. Die „hergebrachten Grundsätze des Berufsbeamtentums", die sich noch im Grundgesetz finden (Art. 33, Abs.5 GG), umfassen Treueverpflichtungen, Alimentationsprinzip (im Unterschied zum Entgelt), Anstellung auf Lebenszeit und Verpflichtung aufs Gemeinwohl jenseits partikularer Interessen. Über den Begriff der außerdienstlichen Führung interessiert sich die Organisation explizit, freilich in historisch wechselnder Akzentuierung, für die "private“ Lebensführung der Beamten.

Personen/Biographien und Stellen in der Organisation werden durch die Laufbahn gekoppelt. Das ermöglicht der Verwaltung die intern flexible Positionsbesetzung aus dem Pool der entsprechend qualifizierten Lebenszeitbeschäftigten. Für die Beamten bedeutet das Laufbahnsystem die Verstetigung und Berechenbarkeit von Karriereerwartungen, wobei ,,jede Beförderung eine Belohnung und zugleich eine Prüfung für die nächste Belohnung“ ist (Wunder 1986: 28). Organisationelle Reibungen zwischen verfügbarem/berechtigtem Personal und Stellen nehmen dann lediglich die Gestalt von Beförderungsstaus an (Mayntz 1985), die Arbeitnehmerrisiken der Entlassung oder des Abstiegs sind bei Wohlverhalten ausgeschaltet.

Die Kriterien für die Rekrutierung geeigneter Beamter entwickeln sich nicht einlinig, etwa von der Herkunft hin zur Qualifikation, wie es für einen Rationalisierungsprozeß staatlicher Bürokratie naheläge. Zwar steigen die Qualifikationsanforderungen an die Beamten, doch setzen die Machtverhältnisse zwischen Herrscher und adligen und bürgerlichen Oberschichten der Verfolgung solcher funktionaler Erfordernisse Grenzen. Beamtenrekrutierung ist politisch insofern, als sie sich nicht allein auf individuelle Leistungsträger beziehen kann, sondern damit die Kooptation bestimmter Standes- und Schichtenvertreter und deren Verpflichtung auf den Herrscher und später den Staat bezweckt.

Waren im 15./16. Jahrhundert mit einem ersten Aufbau staatlicher Zentralverwaltungen (gegen die Interessen des Adels) Kleriker und Juristen bürgerlicher Herkunft aus dem städtischen Patriziat in die Verwaltungen und Gerichte vorgedrungen, so übernehmen in den folgenden Jahrhunderten teilweise wieder Adlige diese Funktionen. Einige Positionen bleiben den ehemals Bürgerlichen erhalten. Aus den akademisch ausgebildeten, bürgerlichen Beamten des 16. Jahrhunderts entwickelt sich so eine ,geschlossene, weitgehend durch Selbstrekrutierung ergänzte Gruppe“" 
(Wunder 1974: 468) von Beamtenfamilien, denen schließlich vielfach ein persönlicher Amtsadel oder auch der erbliche Briefadel verliehen wird.

Im 18. und frühen 19. Jahrhundert wird die Beamtenrekrutierung formalisiert. Schon früher war für die Subalternbediensteten ein praktischer Vorbereitungsdienst mit anschließender Prüfung üblich. Von dort aus konnten praktisch erfahrene Subalternbedienstete ${ }^{6}$ bürgerlicher Herkunft bei guter Leistung und Wohlwollen des Herrschers auch in höhere Verwaltungsämter aufsteigen. In Preußen wird für den höheren Dienst in der Justiz 1755, in der Verwaltung 1770 die mehrstufige Ausbildung mit staatlicher Zugangsprüfung, unbesoldeten Vorbereitungsdiensten und erneuten Prüfungen eingeführt, in den anderen deutschen Ländern zwischen 1770 und 1830.

Zunehmend, und in Preußen definitiv ab 1817 wird das Jurastudium zur Zugangsvoraussetzung. Darauf folgt die Gerichtsauskultatur mit einer Dauer von fünfzehn Monaten bis zu vier Jahren, worauf man sich zum Referendariat bewerben kann. Die Aufnahme liegt im Ermessen des Regierungspräsidenten (Bleek 1972: $123 \mathrm{ff}$.). Damit ist zwar die Beamtenschaft formal höher qualifiziert, doch wird der Bewährungsaufstieg von Subalternbediensteten nunmehr verhindert. Die Geltung des Leistungsprinzips beschränkt sich auf den Zugang zur Beamtenlaufbahn. Die Festanstellung erfolgt dagegen bis in das 20. Jahrhundert nach Anciennität, wobei der Zeitraum von Ausbildung und unbezahlter Arbeit bis zu 20 Jahren dauern kann. ${ }^{7}$

Im Vorfeld totaler Inklusion und Versorgung durch den Staat liegen also für die Beamten lange Phasen der Sozialisation und des Wartens auf eine Stelle. Sie dienen nicht nur dem Wissenserwerb, sondern schließen Unter- und Mittelschichten effektiv von der Beamtenschaft aus und prägen die Lebensführung der Beamten in spezifischer Weise. Solche Lern- und Wartezeiten erfordern und reproduzieren generationsübergreifend ein biographisches Muster aufgeschobener Gratifikation, in welchem Bildung (auch) als Investition gesehen wird. Deutlich wird das etwa an der späten Heirat und geringen Kinderzahl der Beamten. Auch die sparsame, planmäßige Lebens- und Haushaltsfüh-

6 Subalternbedienstete verrichteten den formalen, routinehaften Teil der Verwaltungsarbeit, zu ihnen zählten etwa Sekretäre, Kalkulatoren, Registratoren usw. (Westphalen 1979: 120f.).

7 Ab 1863 mußten etwa Bewerber um ein Referendariat in Preußen nachweisen, zehn Jahre vom eigenen Vermögen leben zu können (Wunder 1977: 365). rung der Beamtenfamilien entspricht sowohl ökonomischer Notwendigkeit als auch ,preußischer Tugend" und bürgerlichem Familienleitbild. ${ }^{8}$

Gerade in der Frühzeit der modernen Bürokratie, im preußischen Beamtenstaat der ersten Hälfte des 19. Jahrhunderts, finden wir ein ethisch-ganzheitlich fundiertes "Passen" von Organisation und Mitgliedern. Die preußischen Beamten personifizieren geradezu die Rationalisierung und Versachlichung, auch wenn man nicht umstandslos von der Selbstdarstellung auf tatsächliche subjektive Dispositionen schließen kann. ${ }^{9} \mathrm{Da}$ ein industrielles Bürgertum erst im Entstehen ist, sehen sich die preußischen Beamten als Hauptagenten und Förderer von Fortschritt und Gewerbefreiheit. Das erfordert mehr als nur regelgeleitetes, pflichtgetreues Vorgehen. Für eine solchermaßen ambitionierte Verwaltung reicht die Leistung bornierter Bürokraten nicht aus. In den Preußischen Geschäftsinstruktionen von 1817 für die Räte und Assessoren heißt es:

„Jeder von ihnen muß den ihm angewiesenen $\mathrm{Ge}$ schäftskreis mit Wärme und innerer Theilnahme auffassen (...) Es ist nicht genug, wenn sie sich blos auf die ihnen zugeschriebenen Sachen beschränken; sie müssen auch aus eigenem Antriebe Gegenstände, welche ihnen nöthig und nützlich scheinen, zur Sprache bringen" (zit. nach Hattenhauer 1980: 202).

Solche Aufmerksamkeit und Eigeninitiative über formal festgelegte Zuständigkeiten hinaus sind Voraussetzung für einen Gestaltungsanspruch der Verwaltung. Hiermit, nicht lediglich mit der eigenen Funktion, sollen sich die Beamten identifizieren.

Während die staatliche Bürokratie die Entwicklung der bürgerlichen Gesellschaft fördert, und dabei die Kontinuität (obrigkeits-)staatlicher Ordnung wahrt, werden für die Beamten ständische Privilegien aktualisiert. Gleichzeitig mit dem Ausbau quasi sozialstaatlicher Absicherung stattet man sie mit Insignien der Aristokratie aus. Die Belohnungen, mit denen die Beamten dem Dienstherren verpflichtet werden, amalgamieren moderne und vormoderne Statuselemente.

8 Vgl. Rosenbaum 1982: 255 ff. Verschuldung gilt als unvereinbar mit korrekter außerdienstlicher Führung der Beamten.

9 Beispielsweise geraten die Wünsche der Ministerien nach einem umfassenden humanistischen Bildungsstudium der Beamten in Konflikt mit einer eher instrumentellen, auf ein baldiges Examen gerichteten Perspektive der Studenten (Bleek 1972: 112). 
Anfang des 19. Jahrhunderts werden, ausgehend von den süddeutschen Staaten, die Beamtenrechte im modernen Sinn ausgestaltet. Beamte erhalten ein Recht auf lebenslange Versorgung, willkürliche Entlassungen werden verboten, auch Hinterbliebene erhalten Pensionen. ${ }^{10}$ Dazu kommt die Ausstattung der höheren Beamten mit adelsanalogen Symbolen wie dem Recht, Uniform (,des Königs Rock") zu tragen, dem Zutritt bei Hofe, dem Recht auf Anrede mit dem Titel und dem Kadettenrecht für die Söhne.

Die neuen Funktionseliten übernehmen so die Position des alten Adels. Die symbolische Kontinuität der Privilegien spiegelt die durchaus erwünschte Selbstrekrutierung der Beamtenschaft (Bleek 1972: $81 \mathrm{f}$.) und nimmt die Beamten von den sozialen Umwälzungen und Freisetzungen der Industrialisierung aus.

Mit der weiteren Differenzierung von Staat und Gesellschaft und der Entstehung einer öffentlichen Meinung wird die politische Zuverlässigkeit der Beamten zum Problem. Studenten und junge Beamte vertreten revolutionäre Ideen von Bürgerrechten und Demokratie, die staatlichem. Handeln Grenzen setzen sollen. Die herkömmlichen Rekrutierungskriterien von Herkunft, Qualifikation und geordneter Lebensführung garantieren der Verwaltung auch bei den Söhnen alter Beamtenfamilien keine hinreichende Loyalität mehr. Politische Zuverlässigkeit wird selbst zum Rekrutierungskriterium. ${ }^{11}$ Politische Überwachung und Kontrolle findet dabei zunächst im sozialisatorischen Vorfeld statt. Die Karlsbader Beschlüsse sehen die Überwachung von Universitäten und Professoren vor, ab 1834 müssen Abitur- und Hochschulzeugnisse auch das „sittliche Betragen“ ausweisen.

Nach 1848 wird politische Opposition zulässig. Die Beamten werden nun auf die Unterstützung der Regierungspolitik verpflichtet. Die Verpflichtung zur standes-/amtsgemäßen außerdienstlichen Führung wird zu politischem Wohlverhalten umdefiniert. ${ }^{12}$ Hebel zur Schließung der Beamtenschaft

10 Vorher hatten die Beamten selbst nach dem Vorbild der protestantischen Geistlichkeit Witwenkassen eingerichtet. In Preußen wurden bis 1888 für die. Hinterbliebenenversorgung Beiträge vom Einkommen der Beamten abgezogen (Wunder 1986: 34).

11 Dabei ubersteigt im 19. Jahrhundert fast durchgängig das Juristenangebot den Bedarf der Verwaltung, so daß eine entprechende Auswahl möglich ist.

12 Vorher richtete sie sich eher gegen Verschuldung, Alkoholismus und sexuelle Fehltritte (Wunder 1986: 88). gegen Demokraten, Freigeister oder gar Sozialisten sind zum einen das Disziplinarrecht, zum andern die fortgesetzte soziale Selektion bei der Rekrutierung. Die erwünschte Herkunft der Bewerber wird hier als ,charakterliche Eignung“ formuliert. Für die Einstellung höherer Beamter werden Reserveoffizierspatent und Mitgliedschaft in studentischen Korps zu Indikatoren der gewünschten rechtsliberalen bis konservativen politischen Einstellung (Hattenhauer 1980).

Bei den Subaltern- und Unterbeamten werden politische Konformität und erwünschter Habitus dadurch erreicht, daß man sie seit dem 18. Jahrhundert aus der Gruppe der Unteroffiziere und Militärveteranen rekrutiert. ${ }^{13}$ Die Ausweitung der Beamtenprivilegien auf die Gruppe der Subalternen und Unterbeamten bindet auch sie in den Tausch von Treue gegen Fürsorge ein. Zu einem Zeitpunkt, als die Bismarcksche Sozialversicherung Arbeiter abzusichern beginnt, wird der Abstand zwischen ihnen und den unteren Beamten dienstrechtlich befestigt und die Interessenwahrnehmung der Beamten disziplinarisch begrenzt.

Indem die staatliche Verwaltung über die Aktualisierung ständischer Inklusionsmodi die Beamten vom gesellschaftlichen Wandel abkoppelt, verschiebt sich das Gewicht bei Rekrutierung und Erscheinungsbild der Beamten vom selbstbewußten, aufgeklärten, aber durchaus autoritären preußischen Staatsdiener, der Freiheit und Fortschritt bei seinem Stand am besten aufgehoben findet, hin zu dem „Bürgerlichen mit feudalen Prätensionen“, wie Max Weber die schneidig-arroganten, konservativen Assessoren des Kaiserreichs charakterisiert (Weber 1988: 389 ff.). Die Korpsstudenten, zukünftige Beamte mit Stiefeln, Säbeln und Duellen, inszenieren diesen gesellschaftlicher Rationalisierung gerade entgegenlaufenden Inklusionsmodus noch einmal.

Stichworte für die Verknüpfung von Organisation und Biographie bei den Beamten sind also Sozialisation, soziale Selektion bei der Rekrutierung, Laufbahn- und Lebenszeitprinzip. Letztere gewährleisten die Absicherung und Versorgung der Staatsdiener in der Organisation (über die Dauer eines Vertrages hinaus) und belohnen das gewünschte commitment mit berechenbaren Perspektiven. Die Gestaltung des Zugangs zur Organisationsmitgliedschaft selbst selektiert die ge-

13 Umgekehrt wird mit der Aussicht auf den „Zivilverwendungsschein", d.h. eine Anstellung als Beamter die neunjährige Verpflichtung als Unteroffizier attraktiver. 
wünschten Organisationsmitglieder. Dabei nehmen die Verwaltungen die Herkunftsfamilien der Kandidaten bis zur Festanstellung in die Pflicht. Danach dehnen sie ihre Fürsorge auch auf die Familien der Beamten aus. So entstehen generationsübergreifende Bindungen und Aufstiegserwartungen - die Söhne der Subalternbeamten studieren möglicherweise und werden höhere Beamte (Bleek 1972). Über den engen Rahmen der Mitgliedsrolle hinaus weisen auch die Probleme politischer Zuverlässigkeit und außerdienstlicher Führung. Die Verwaltung schafft sich also bis hin zur Festanstellung mehrere Sicherheitsnetze von der „richtigen“ Herkunft über die außer- und innerorganisatorische Sozialisation bis zu Prüfungen und Ermessensentscheidungen über den weiteren Aufstieg.

\section{Rationalisierte Frauen: Das Fräulein vom Amt}

Komplementär zur Inklusion als Familienernährer bei den männlichen Beamten (über Alimentationsprinzip und Hinterbliebenenversorgung) ${ }^{14}$ schließt die Verwaltung unerwünschte Versorgungsrisiken wiederum aus. Für niedere Zivilbedienstete und Unterbeamte gelten teils bis ins 20 . Jahrhundert ${ }^{15}$ Heiratsverbote oder Erlaubnisvorbehalte, die ihre Tradition in den ständischen Heiratsverboten für Gesellen und Gesinde haben (Wunder 1986: 34). Hier knüpft - mit wesentlich anderen Wirkungen - der Zölibat der weiblichen Beamten an. ${ }^{16}$ Er setzt für diese Frauen das moderne Modell der Übergangsberufstätigkeit durch.

Frauen werden seit etwa dem letzten Drittel des 19. Jahrhunderts als Lehrerinnen oder (selten) Ärztinnen in den höheren Dienst und bei Post bzw. Bahn in untergeordnete Tätigkeiten eingestellt. Am Beispiel des „Fräuleins vom Amt" soll die Entwicklung hin zu einem eher „exklusiven“

14 Hausen (1980: 175) sieht in den Familien der höheren Beamten die Trennung von Beruf und Haushalt und damit das bürgerliche Hausfrauen/Ernährermodell zuerst und am intensivsten durchgesetzt.

15 Anfang des 20. Jahrhunderts erhielt nur etwa jeder zehnte Postillion des städtischen Poststalls München eine Heiratserlaubnis (Gold 1993: 25).

16 Der Ausdruck „Zölibat“ mit seinen geistlichen Konnotationen wird in der zeitgenössischen Literatur allgemein verwendet.
Zusammenhang von Organisation und Lebensführung verdeutlicht werden. ${ }^{17}$

Die Deutsche Reichspost stellt seit 1887 zunächst probeweise, dann ab 1891 regulär Frauen für die Telefonvermittlung ein. Sie bilden die am schnellsten wachsende Gruppe der Beamtinnen. Begründet wird die Beschäftigung von Frauen nach außen vor allem mit Eignungsargumenten wie ihrer Stimmlage und Sozialkompetenz, nach innen mit Kosteneinsparungen. Der Unterstaatssekretär Fischer meint 1894 im Reichstag, daß „der Teilnehmer friedlich wird, wenn ihm aus dem Telephon eine Frauenstimme entgegentönt" (zit. nach Wagner 1913: 76).

Der Hauptgrund für die Beschäftigung von Frauen ist jedoch, daß die neue Aufgabe nicht in die Ordnung von Hierarchie und Laufbahn der männlichen Postbeamten paßt (Holtgrewe 1989). Der Personalbedarf in der Telefonvermittlung steigt stärker, als daß diese Tätigkeit in die Laufbahnen unterer oder mittlerer männlicher Beamter einzubauen wäre; die Arbeit verlangt mehr Routine, als von übergangsweise dort beschäftigten Beamten erworben werden kann; schließlich reichen Allgemeinbildung und Sprachkompetenz von Unterbeamten oder Hilfskräften nicht aus. Eine Segmentierung der Organisationsstruktur bietet sich also an. Indem Frauen beschäftigt werden, wird der Telefonvermittlungsdienst vom normalen, männlichen Laufbahnsystem abgeschottet.

Als Fernsprechgehilfinnen werden ledige Frauen und kinderlose Witwen zwischen 18 und 30 Jahren eingestellt, mit einer Schulbildung oberhalb der Volksschule. Sie werden zwischen zwei und sechs Monaten ausgebildet, arbeiten dann diätarisch gegen Tagegelder und werden schließlich nach etwa neun Jahren planmäßig angestellt. ${ }^{18}$ Auch für sie gelten also die typischen langen Wartezeiten bis zum vollen Beamtenstatus.

Das bedeutet jedoch, anders als bei ihren männlichen Kollegen, nicht einen zeitlichen Aufschub bis schließlich der Familienernährerstatus die Festan-

17 Die Aussagen über Berufsbiographien der Beamtinnen stützen sich auf Forschungen, die Ursula Holtgrewe 1989/90 für das Postmuseum Nürnberg durchführte. In diesem Zusammenhang wurden u.a. Lebensläufe aus Personalakten rekonstruiert.

18 Bei Frauen meint planmäßige Anstellung nicht Anstellung auf Lebenszeit, die Verwaltung behält sich eine dreimonatige Kündigungszeit vor. Begründet wird das mit der Möglichkeit einer unehelichen Schwangerschaft (Wagner 1913: $34 \mathrm{f}$.). 
stellung abrundet. Vielmehr rekonstruiert die Post die doppelte Rationalisierung des Lebens und Arbeitens von Frauen ${ }^{19}$ als direktes Entweder-Oder: Ehe und Beamtinnenstatus schließen sich aus, mit der Heirat endet das Dienstverhältnis. ${ }^{20}$

Auf diese Art werden zwei biographische Normalmodelle möglich. Der Lebensberuf bei der Post kann eine Alternative zur Ehe sein. Er bietet immerhin nach zehn Jahren einen sicheren Arbeitsplatz mit Gehaltssteigerungen nach Dienstalter und Pensionsberechtigung. Sonst bleibt die Möglichkeit der Übergangsberufstätigkeit bis zur Heirat. 1914 waren $22,3 \%$ der 35.668 Frauen bei der deutschen Post planmäßige, pensionsberechtigte Beamtinnen (Nienhaus 1993b: 73). ${ }^{21}$

Der Zwang zur Entscheidung zwischen Lebensberuf und Übergangsberufstätigkeit erweist seine Funktionalität für die Post aufgrund des hohen gesundheitlichen Verschleißes der Vermittlungsbeamtinnen. Die "natürliche" Fluktuation durch Heirat hat den Vorteil, Personalkosten, Krankenstände und Versorgungsansprüche zu minimieren. $^{22}$

Eine solche Externalisierung von Risiken, legitimiert durch den Verweis auf weiblich-körperliche Besonderheit, strukturiert das Inklusionsverhältnis von Frauen bei der Post insgesamt. Die Verwaltung und ihre Vertrauensärzte schreiben die hohen Krankenstände und die Folgen von Arbeitsunfäl-

19 Frauen des Bürgertums und Kleinbürgertums betrifft gesellschaftliche Rationalisierung in doppelter Weise. Als Berufssphäre und Privatheit auseinandertreten, entsteht die „Nur-Hausfrau“, die das männliche Normalmodell der Erwerbstätigkeit durch sparsame Haushaltsführung und kultivierte Häuslichkeit unterstützt. Aus den verkleinerten Familien aber werden auch Frauen freigesetzt: Mit der Industrialisierung produktiver Arbeiten sind unverheiratete Töchter und Schwestern nicht mehr in den Haushalten zu beschäftigen. Die Frauenbewegung klagt ihre Teilhabe an der neuen Gesellschaft ein. Die Erschließung standesgemäßer Berufe oberhalb von Fabrikarbeit steht seit den 1860er Jahren als „Jungfern-“ oder „Tantenfrage“ auf der politischen Tagesordnung (Twellmann 1993). Die Rekrutierung neuer, weiblicher Arbeitskräfte ist somit eng mit Öffnungen, Bewegungen, auch Emanzipationsprozessen in Frauenbiographien verbunden.

20 Vgl. Wagner 1913: 37 f. und für Bayern Antrag 1895.

21 Auch Braun schätzt, daß etwa $20 \%$ der Beamtinnen sich für die lebenslange Beschäftigung entscheiden (1912: 21).

22 Ob die Aufgabe des Berufs bei den Frauen der Grund zur Heirat oder eine wenig erwünschte Nebenwirkung war, ist hier nicht zu erschließen. len in der Telefonvermittlung ${ }^{23}$ einer Veranlagung der Frauen zur Hysterie zu, nicht den spezifischen Belastungen ihrer Arbeit. Einstellungs- und Nachfolgeuntersuchungen sollen potentiell hysterisch veranlagte Frauen vor der Festanstellung identifizieren, um sie ggf. zu entlassen.

Die Wartezeit bis zur Festanstellung ist also für die Frauen Bewährungsphase vor allem auf gesundheitliche Robustheit hin. „Vater Staat“ dagegen wartet mit der Gewährung der Beamtinnenprivilegien $a b$, ob sich nicht doch ein anderer Ernährer findet. Er tritt sozusagen erst subsidiär in seine Verpflichtungen ein.

Auch die Einstellungskriterien verschieben sich entsprechend von Bildung und bürgerlicher Respektabilität hin zu Gesundheit und psychischer Stabilität. Die Kandidatinnen legen eine Prüfung in Deutsch, Rechnen und Geographie ab, dazu kommt eine ärztliche Untersuchung. Bewerberinnen müssen bei den Eltern wohnen oder, so das Weisungsbuch der Umschaltestelle in Nürnberg, „sich über den Anschluß an eine achtbare Familie ausweisen“ (Weisungen 1885-1917: 13).

Über den Eindruck sozialer Kontrolle und paternalistischer Fürsorge hinaus deutet sich hier ein personalpolitisches Problem der Post an. Bei Frauen verflüssigen sich die traditionellen Herkunfts- und Rekrutierungskriterien für Beamte, denn weiblichen Lebensläufen ist die Eignung nicht anhand von institutionalisierter Schulbildung oder gar militärischen Zeugnissen zu entnehmen. Die jungen Frauen des (Klein-)Bürgertums, die die Post rekrutieren will, sind dem Leitbild nach in ihren Erfahrungen auf die häusliche Sphäre beschränkt. Also rekurriert die Post auf Indikatoren ordentlicher Familienverhältnisse, beurteilt die Frauen in ihrer Eigenschaft als „Töchter“. Vorherige berufliche Erfahrungen sind dann eher suspekt:

„Eine vorsichtige Prüfung über das Vorleben wird besonders bei solchen Personen vorgenommen, die bereits in einem andern Berufe tätig gewesen sind, um festzustellen, ob sich aus ihrer bisherigen

23 Ursachen waren Blitzschläge, aber auch rücksichtsloses Verhalten der Teilnehmer. Ehe die Telefone durch Zentralbatterien in den Ämtern mit Strom versorgt wurden, mußte man, um das Amt zu rufen, die nötige Spannung selber durch Kurbeln erzeugen. Hatte sich nun die Telefonistin schon eingeschaltet, „bekam sie Strom“, wie es im Jargon hieß, einen akustisch-elektrischen Schock, der neurologische Folgen von Zittern oder Kopfschmerzen bis zu Lähmungserscheinungen haben konnte (vgl. Milles 1993). 
Beschäftigung Bedenken gegen die Aufnahme im Beamtendienst ergeben" (Wagner 1913: 23).

Faktisch wechseln viele Frauen nicht direkt aus der Familie zur Beschäftigung bei „Vater Staat“. Wie aus den Personalakten ersichtlich ist, modernisieren sie vielmehr ihre Arbeitsverhältnisse. Sie haben das Lehrerinnenexamen, feine Näharbeiten in Heimarbeit gemacht, als Gouvernanten (zum Teil im Ausland) oder als Büroangestellte gearbeitet. Oftmals müssen kranke Angehörige unterstützt werden. Für diese Frauen bedeutet die Arbeit bei der Post den Schritt von quasi traditionalen, persönlich-grenzenlosen familialen oder kleinbetrieblichen Arbeitsverhältnissen zu klaren $\mathrm{Zu}$ ständigkeiten, geregelten Arbeitszeiten, Zusammenarbeit mit Kolleginnen.

Auch die Arbeitsanforderungen im Zentrum moderner Kommunikationstechnik kontrastieren mit den von der Post zunächst gesuchten Qualifikationen. Bürgerlich-wohlanständiger Habitus, sprachliche Kompetenz und Fingerfertigkeit reichen, wie sich herausstellt, nicht aus. Mehrfach-Aufmerksamkeit, Konzentrationsfähigkeit und Nervenstärke erweisen sich als notwendig für die Vermittlungsarbeit an der störungs- und konfusionsanfälligen Technik. Das sind Fähigkeiten, für die die Rekrutierung nach (klein-)bürgerlicher Herkunft nicht unbedingt garantiert. ${ }^{24}$

Aus Verweisen und Rechtfertigungen in den Personalakten (s. Fußnote 17) kann man schließen, $\mathrm{da} \beta$ gemessen an den Arbeitsanforderungen ,gute" Vermittlungsbeamtinnen selbstbewußtere und weniger fügsame Frauen waren, als Vorgesetzte das für wünschenswert hielten. Es finden sich Disziplinierungen für verschiedenste Regelverstöße, von freundschaftlichen Umarmungen bis zu offensolidarischer Unterstützung von Kolleginnen, denen die Entlassung wegen „unsittlichen Verkehrs mit einem Manne" drohte, ${ }^{25}$ so daß sich auf vielfältige informelle Auseinandersetzungen im Arbeitsalltag schließen läßt.

Eine Arbeit anzunehmen, die wenig mit traditionellen weiblichen Beschäftigungen, dafür aber mit neuer Technik und neuartigen Kommunikationsformen zu tun hat, hat sehr wahrscheinlich auf Seiten der Subjekte Enttraditionalisierungs- und

24 Die extrafunktionale Qualifikation sozialer Kompetenz wird dagegen durch die Reglementierung der Arbeit ersetzt. Sollten ursprünglich die Frauen vom Amt erzürnte Teilnehmer beruhigen und freundlich stimmen, so werden die amtlichen Redewendungen schließlich doch detailliert vorgegeben.

25 Personalakte Käthe E., Postmuseum Nürnberg.
Emanzipationsprozesse schon zur Voraussetzung, die dann wieder befördert werden. Die Frauen machen neue Arbeitserfahrungen, können die Anforderungen auch als eigene Kompetenz erleben. Ihre Leistungen sind für die Post dann nicht ohne Aufmüpfigkeit und Solidarisierungen zu haben. Die angeworbenen Arbeitskräfte erweisen sich als durchaus eigensinnig.

Die Post kann also letztlich nicht auf traditionelle Weiblichkeitsbilder rekurrieren. Vielmehr nehmen sich Übergangsberufstätigkeit, Lockerung der traditionell-umfassenden Fürsorge für die Beamten und Minimierung von Risiken und Ansprüchen moderner aus, als vor der Folie des männlichen Berufsbeamtentums zu erwarten gewesen wäre. Frauen in der Organisation verschieben die Grenzen dessen, was inkludiert wird, bzw. die „Risiken“ der Weiblichkeit werden gerade ausgeschlossen. Dennoch resultiert diese Modernität nicht allein aus vorgeplantem patriarchalem Kalkül. Zwar schafft sich die Post eine überqualifizierte, fluktuierende Randbelegschaft für verschleißintensive Massenarbeiten (Nienhaus 1993b). Zunächst jedoch besteht Ungewißheit über Anforderungen, funktionale und extrafunktionale Qualifikationen der neuen Beschäftigten. Der Rückgriff auf (klein-)bürgerliche Reputierlichkeit reicht nicht aus. Es sind nicht bürgerliche „Nur-Töchter“, die weiblich-bescheiden die zugewiesenen Arbeitsplätze an den Klappenschränken einnehmen. Kompetente Aufgabenerfüllung für die Post ist nicht ohne gewisse Emanzipationschancen und Eigensinn der Frauen zu bekommen.

Für sie öffnen sich neue biographische Alternativen und Freiräume. Sie ergreifen die Gelegenheit, ihre Arbeitsverhältnisse zu modernisieren und setzen sich für erweiterte berufliche Spielräume ein. ${ }^{26}$ Die mit dem Beamtenstatus verbundenen Fürsorgeversprechen klagen sie gegen ihre nur marginale Inklusion ein. Selbst die erzwungene Alternative von Beruf und Ehe, die Frauen am Rand der Organisation hält, eröffnet immerhin eine biographische Option mehr.

26 Ab 1912 nahm der als Dachverband verschiedener örtlicher Vereine gegründete Verband der Reichs-Postund Telegraphenbeamtinnen die Interessen der Beamtinnen wahr. Er neigte naheliegenderweise eher zum Modell des Lebensberufs und forderte den Ausbau der Laufbahn für Frauen, bessere Besoldung und Gesundheitsschutz, strebte also eine stärkere Professionalisierung an. Dazu kamen vielfältige Aktivitäten der Selbsthilfe (vgl. Nienhaus 1990, Koch 1993). 


\section{Zeitarbeit}

Aus der neueren Entwicklung der Beziehung von Organisation und Biographie wollen wir nun zunächst auf ein Beispiel zurückgreifen, in dem das Problem der Unbestimmtheit des Arbeitsvertrages auf spezifische Weise gelöst wird und eine tatsächlich rein vertragliche Beziehung das Arbeitsverhältnis prägt: das Beispiel der Zeit- bzw. Leiharbeit. Dies gelingt u.a. dadurch, daß das Arbeitsverhältnis und das Beschäftigungsverhältnis entkoppelt werden. Zeitarbeitnehmer werden von "Verleihunternehmen" grundsätzlich unbefristet eingestellt und zu „befristeten Einsätzen“ an die Nutzerunternehmen bzw. Einsatzbetriebe verliehen. Dies ist durch das „Arbeitnehmer-Überlassungsgesetz" geregelt. Durch diese „Triangulation" zwischen Arbeitnehmer, Nutzerunternehmen und Verleihunternehmen, werden die Bindungseffekte und Risiken, die in einem auf Dauer angelegten „Normalarbeitsverhältnis“ bestehen, anders verteilt als beim normalen Arbeitsvertrag (Brose et al. 1990). Der normale Arbeitsvertrag ist in der Regel zeitlich unbefristet und inhaltlich unterbestimmt. Es handelt sich eben nicht um einen spezifischen Werkvertrag, in dem genau festgelegt wird, welche Leistung zu erbringen ist. Der normale Arbeitsvertrag ist dagegen insofern ein „Mietvertrag besonderer Art" (Dragendorf/Heering 1987), als das gemietete Arbeitsvermögen nicht von der Person seines Trägers abgekoppelt werden kann. Ganz anders beim Zeitarbeitsvertrag.

Das Nutzerunternehmen - in dem die Arbeit verrichtet, die Dienstleistung erbracht werden soll fordert von dem Verleihunternehmen ein in der Sachdimension weitgehend spezifiziertes Arbeitsvermögen an: beispielsweise einen Facharbeiter einer bestimmten Qualifikation; einen Ingenieur mit besonderen Spezialkenntnissen. „Arbeit nach $M a B$ " heißt das in der Marketingstrategie der Zeitarbeitsunternehmen. Wird eine von dem Verleihunternehmen entsandte Person den qualifikatorischen Anforderungen - in welcher Hinsicht auch immer - nicht gerecht, wird sie ausgetauscht. Für das Nutzerunternehmen entfallen also die Selektions- und Sozialisationsprozesse, die ein unspezifisches Arbeitsvermögen in ein betrieblich nutzbares Arbeitsvermögen umformen. Die Kopplung von Arbeitsvermögen und der Person ihres Trägers wird dadurch aufgehoben - die Personen sind austauschbar. Die Such- und Selektionsprozesse sowie die entsprechenden Kosten - fallen also nicht beim Nutzerunternehmen an, sondern beim Verleihunternehmen, das diese aufgrund seiner
Zwischenstellung zwischen dem Arbeitnehmer und dem Nutzerunternehmen in spezifischer Weise organisiert. Das geschieht etwa, indem bei der Einstellungsselektion Qualifikationen primär unter dem Gesichtspunkt ihrer vielseitigen Verwendbarkeit (Generalqualifikationen) geprüft und in Einsätzen erprobt bzw. getestet werden.

So unspezifisch also die soziale Dimension des Zeitarbeitsvertrages ist - der jeweilige Vertragspartner ist austauschbar - so spezifisch ist er in der Sach- und in der Zeitdimension. Aus dem in der Sachdimension unbestimmten Arbeitsvertrag wird - in der Zeitarbeit - zunehmend ein Zuliefer- und Werkvertrag, der die Besonderheit des Arbeitsvertrages abstreift (Streeck 1988). In der Zeitdimension werden durch die Ausdifferenzierung von Beschäftigungsverhältnis (mit dem Zeitarbeitsunternehmen) und dem Arbeitsverhältnis (am Einsatzort) grundsätzlich zwei Zeitperspektiven geschaffen. Eine Betriebszugehörigkeit zum Zeitarbeitsunternehmen von grundsätzlich unbefristeter Dauer, in der Regel jedoch von mehreren Monaten bis einigen Jahren, ist mit vielen kürzeren Einsätzen in Nutzerunternehmen verbunden. Diese Ausdifferenzierung macht aus dem jeweiligen Einsatz des Arbeitsvermögens im Entleihbetrieb ein „Ereignis“, das in seiner Dauer begrenzt ist. Anschlußprobleme aus der Vergangenheit und in die Zukunft gibt es aus der Organisationsperspektive des Einsatzbetriebes nicht.

Der Beschäftigungsvertrag zwischen dem Zeitarbeitsunternehmen und dem Einsatzbetrieb löst dieses Problem der Transformation von Zeitperspektiven auf der Ebene von zwischenorganisatorischen Vertragsbeziehungen. Man spricht hier auch von Fristentransformation. Die längerfristigen Bindungen an einen ,Träger des Arbeitsvermögens" werden so - für den Einsatzbetrieb - gekappt. Für das Zeitarbeitsunternehmen werden sie nicht in der gleichen Weise wirksam, wie für einen Beschäftiger im „Normalarbeitsverhältnis“. Aus der Sicht der Beschäftigten wird damit die längerfristige biographische Perspektive in einen Möglichkeitsraum zwischen den Organisationen verwiesen. Anschlüsse, Sinnzusammenhänge werden so über befristete Inklusionen, Diskontinuitäten hinweg von ihnen selbst herzustellen sein. Eine solche befristete Inklusion von Mitgliedern entlastet die Organisation von Rücksichten auf soziale Bindungen.

Umgekehrt sind die Beschäftigten - im Hinblick auf ihre Motivation, auf ihre Biographie, ihre sozialen Bindungen, eben auch „frei-gestellt“. So kön- 
nen unterschiedliche Sozialisationsprozesse, Biographien und Motive an die Organisation ,angeschlossen" werden. Und dies ist in der Tat der Fall. Wie wir in unserer Untersuchung (Brose et al. 1993) zeigen konnten, sind die (berufs-)biographischen Entwicklungsmuster oder Weisen der Lebensführung, die man in der Zeitarbeit vorfindet, sehr vielgestaltig. Für alle Beschäftigten gilt zwar, daß sie in einem Normalarbeitsverhältnis entweder nicht arbeiten können oder nicht arbeiten wollen. Doch vor dem Hintergrund dieser Gemeinsamkeit gibt es sehr unterschiedliche biographische Profile. Sie reichen um es sehr klischeehaft auszudrücken - von dem Arbeitnehmer, der die Diskontinuität seiner Arbeitsverhältnisse als Teil seines „flexiblen Lebensstils" verstanden wissen will bis zum Arbeitnehmer, der in der Zeitarbeit eine vorletzte Chance für die Integration in das Beschäftigungssystem sieht und diese nutzt. Sie reichen andererseits von dem hochgradig individualisierten Arbeitnehmer, der in einem Singlehaushalt lebt, bis zu dem traditionellen, familienorientierten Arbeitsemigranten, dessen Lebensführung an stabilen sozial-moralischen Milieus orientiert ist. All diese biographischen Entwicklungsmuster können in der Konstruktion der Zeitarbeit an die Organisationen angeschlossen werden. Das Vorleben und die Herkunft spielen dabei im Grundsatz ebensowenig eine Rolle, wie die Zukunftsperspektive der Personen. So kann es dann auch zu Konstellationen fast schon anekdotischer Art kommen, die einen ehemals wegen schweren Raubes vorbestraften, auf Bewährung entlassenen Häftling zum Einsatz in einem Unternehmen für Sicherheitstransporte kommen lassen.

Grundsätzlich gilt also, daß mit der Nutzerorganisation völlig unterschiedliche biographische Entwicklungsmuster der eingesetzten Zeitarbeitnehmer kompatibel sind. Andererseits sind Formen der Ko-Evolution von Mustern biographischer Entwicklung, wie wir sie in der Zeitarbeit gefunden haben, und der Entwicklung eines neuartigen Organisationstypus möglich. Dabei denken wir insbesondere an solche Muster biographischer Entwicklung, deren Sinnstruktur sich mit der Struktur von Organisationen des Typs ,ad-hocracy" (Mintzberg 1983) ergänzt bzw. in einen wechselseitigen Steigerungsverhältnis entwickelt. Dabei handelt es sich um Organisationen, die für wechselnde Ziele in turbulenten Umwelten hohe Motivations- und Einsatzressourcen von Mitarbeitern abschöpfen, ohne dabei über längerfristige Bindungsmechanismen deren commitments ein- werben zu können. Organisationen diesen Typs nehmen in ihrer Bedeutung $z u,{ }^{27}$ und es ist sicher sinnvoll anzunehmen, daß solche Organisationen Mitglieder rekrutieren, deren Lebensführung und Biographie sich in einem Verhältnis der Synergie zu diesem Typus entwickelt.

Um es zu wiederholen: Die Inklusionsform der Zeitarbeit erlaubt der Organisation, meist in dafür entsprechend ausdifferenzierten lokalen Zonen, die Nutzung unterschiedlichster biographischer Ressourcen: chronisch Unruhige oder periodisch rebellische Personen; ehemals vielversprechende, nun aber ausgebrannte Karrieristen; Desillusionierte, die sich in Weiterbildungs- und Warteschleifen bewegen, ohne vorwärts $\mathrm{zu}$ kommen; ambitionierte Job-hopper, aber auch genialische Quartalssäufer. Sie alle sind in formalen Organisationen potentielle Ursachen für Irritationen, Konflikte. In der „Arbeit auf Zeit" können sie und die Organisation durch die wechselseitig genutzte Indifferenz miteinander ko-existieren.

Wenn es aber um Ko-Evolution geht, dann sind solche Kombinationen von Indifferenzen vermutlich nicht ausreichend. Hier müssen die $\mathrm{Mu}$ ster biographischer Entwicklung und die Organisationsentwicklung in positiver Rückkopplung immer wieder neu aneinander anschließen können. In ad-hocracies würden dann etwa Muster biographischer Entwicklung passen, die weder durch kontinuierliche, teleologische Orientierungen noch durch fatale Entwicklungen geprägt sind, die vielmehr die Erfahrung von Brüchen und Diskontinuitäten gemacht und so verarbeitet haben, daß daraus nicht nur Anspruchsreduktion, sondern auch Ambiguitätstoleranz resultiert; bei denen die Herauslösung aus dem Herkunftsmilieu - obwohl sie nicht zur Integration in neue Milieus geführt hat - zum Aufbau eigensinniger und (teilweise) multipel strukturierter Lebensformen geführt hat.

\section{Betriebsclans}

Vor dem Hintergrund der zuletzt vorgestellten Inklusionsverhältnisse nimmt der Clan eine eigentümliche Kontraststellung ein. Während wir dort Linien nachzeichneten, die zur Partial- bzw. Tangentialinklusion führen, möchten wir nun am Bei-

27 Es sind dies z. B. Formen befristeter inner- und zwischenbetrieblicher Kooperation, etwa in Projektteams oder Unternehmensnetzwerken. 
spiel der Betriebsclans wiederum eine Tendenz hin zur Totalinklusion aufzeigen. ${ }^{28}$

Der Clanbegriff „,bezeichnet einen bestimmten Typus der (selbstregulierenden, kaum formalisierten, d.A.) Kooperation, der auf einem hohen Grad gemeinsamer Sozialisation der Beteiligten beruht " (Deutschmann 1987: 146). Der Begriff selbst geht auf die Arbeiten William G. Ouchis zurück (1980; 1981; Ouchi/Jaeger 1978), der unter Clans ,intimate associations of people engaged in economic activity but tied together in a variety of bonds" versteht (1981: 70).

Ouchi konzipiert seinen Clanbegriff in Anlehnung an den Durkheimschen. Nach Durkheim stellen Clans organische Verbindungen dar, die Verwandtschaftsbeziehungen ähnlich sind, aber nicht unbedingt auf Blutsverwandtschaft gründen müssen (1977: 216 ff.). Im Anschluß daran stellt Ouchi die These auf, daß es genau dieses fiktive, nichts desto trotz bindende Verwandtschaftsverhältnis ist, das die Regulierung von Kooperationsbeziehungen in vielerlei Hinsicht entlastet (1980: 90). Das verwandtschaftsähnliche Verhältnis bzw. das dahinterstehende einende Band organischer Solidarität ebnet nämlich die Differenz zwischen eigenen und fremden Interessen ein. Mithin ziehen alle an einem Strang und kommen dabei ohne explizite Regeln oder andere flexibilitätseinschränkende Formalia und entsprechende Kontrolleinrichtungen aus.

Weil es Clans gelingt, das Kooperationshemmnis schlechthin, nämlich interne Interessendivergenzen, ein für alle mal aus dem Weg zu räumen, sind sie - so Ouchi - das Ideal einer vollständig flexiblen, hoch effizienten und perfekt integrierten Organisation (1981: 90). Sie zeichnen sich durch eine flexible Positionsbesetzung auf der Grundlage diffus-entgrenzter Mitgliedschaftsverhältnisse aus. Enge Kopplungen zwischen Statushierarchie und funktionaler Aufgabenverteilung sind den Clans genauso fremd wie formal geregelte Kompetenzstrukturen oder scharf geschnittene Kommunikationslinien und Weisungsbefugnisse. Unter diesem Aspekt setzen sich Clans deutlich von den klassischen Konzepten der Organisationssoziologie ab, die gerade in der Formalisierung von Mitglied-

28 Um ein Mißverständnis zu vermeiden, zu dem womöglich die Materie selbst einlädt, sei im Vorgriff betont, daß wir unter Totalinklusion nicht umfassende Kontrolle in totalen Institutionen verstehen. Es geht vielmehr um einen bestimmten Typus von Mitgliedschaftsverhältnissen, dessen zentrales Bestimmungsmoment das der Entgrenzung ist. schaftsverhältnissen und der damit verbundenen Ausblendung von Subjektivität den Garanten organisatorischer Effizienz erblicken. Clans hingegen zapfen bewußt die lebensweltlichen Ressourcen ihrer Mitglieder an und gestalten sie mit dem Ziel um, solche Potentiale wie z. B. Vertrauen, Intimität und Loyalität systematisch in den Dienst der Organisation zu stellen. Dazu bedarf es einer bestimmten subjektiven Disposition der Mitglieder, die durch eine spezifische Verklammerung der Personalrekrutierung mit organisationsinternen Sozialisationsprozessen nachgefragt, entwickelt und gefestigt wird. Diese These soll nun am Beispiel der Einstellungspolitik japanischer Unternehmen entwickelt werden. ${ }^{29}$

Das Clanmodell fußt auf einer Passung zwischen nicht mehr exakt spezifizierbaren Erwartungen der Organisation einerseits und (psychisch) plastischen, fachlich qualifizierten Funktionsträgern andererseits, die sich zudem optimal in die homogene Personalstruktur einfügen sollen. Entsprechend weit ist der Kriterienkatalog gefaßt, der bei der Personalrekrutierung zum Zug kommt.

Bei der Einstellung neuer Mitglieder kommt der Ausbildung bzw. der Art des Bildungspatentes ein hoher Stellenwert zu. Die Großunternehmen rekrutieren ihre Nachwuchskräfte prinzipiell nur an ausgewählten Schulen bzw. den Universitäten, welche die in Japan sehr etablierten Rankinglisten unangefochten seit Jahr und Tag anführen. Da die Spitzenuniversitäten hinsichtlich der Aufnahmebedingungen und des erfolgreichen Studienabschlusses hochselektiv verfahren, gilt die Kompetenz ihrer Studenten als gesichert.

Um an einer der begehrten Eliteuniversitäten überhaupt angenommen $\mathrm{zu}$ werden, müssen die jungen Japaner neben Intelligenz vor allem auch Leistungsbereitschaft, hohe Motivation und Diszi-

29 Der eigentlichen Diskussion seien noch zwei kurze Hinweise vorangestellt: 1) Gegenwärtig wird das Clanmodell vor allem mit Blick auf japanische Unternehmen diskutiert. Der Clan selbst hat allerdings seine Vorläufer in amerikanischen Managementkonzepten. Diese wurden in Japan in den 20er Jahren unseres Jahrhunderts aufgegriffen, als sich die japanische $\mathrm{Ge}$ sellschaft amerikanischen Einflüssen und Ideen öffnete. Folglich handelt es sich bei den Betriebsclans nicht um ein originär japanisches Phänomen - es kehrt vielmehr versehen mit einer spezifisch japanischen kulturellen Aufladung und Perfektionierung in seinen ursprünglichen Kulturkreis zurück. 2) Empirisch ist die clanförmige Gestaltung von Mitgliedschaftsverhältnissen auf den Kreis der regulär, sprich lebenslang Beschäftigten japanischer Großunternehmen beschränkt. 
plin mitbringen (Yoshime/Schönbauer 1994: $56 \mathrm{f}$.). Diese Grundtugenden werden im Verlauf der Ausbildung weiter gefördert und gefestigt. Die Elitenrekrutierung bietet den Unternehmen also den Vorteil, da $B$ sie auf ideal vorsozialisierte Bewerber treffen. Ideal insofern, als die Bewerber mit dem erfolgreichen Abschluß ihres Studiums nicht nur kognitive Leistungsfähigkeit unter Beweis gestellt haben, sondern auf dem Weg dahin auch die Kardinaltugend schlechthin, nämlich disziplinierte Selbstkontrolle, verinnerlicht haben. ${ }^{30}$

Weiterhin zeichnen sich die Absolventen der Eliteuniversitäten dadurch aus, daß sie über einen gemeinsamen sozialisatorischen Hintergrund verfügen, der als "Korpsgeist" in der Firma fortgeschrieben wird. So sind die Neuzugänge mit den anderen Firmenmitgliedern gewissermaßen von vornherein durch das Band der geteilten Sozialisationsgeschichte geeint (Deutschmann 1987: 136).

Mindestens genauso wichtig, wenn nicht gar bei weitem wichtiger als die Ausbildung ist die psychische Plastizität des Bewerbers.

Dazu ein Personalchef: „What we look for is 'raw materials'. We do not want students who have previous job-related experience. If we hire a student with moulding experience, we first have to break him of acquired work habits and re-train him so that he would adjust to our company color" (zit. nach Ishida 1986: 169). ${ }^{31}$

Dieser Grundtenor spiegelt sich auch in der Selbstdarstellung der Bewerber wider. So berichtet Clark, daß sich die Bewerber selbst als "halfformed creatures“ präsentieren, „waiting for company membership to make them whole" (Clark 1979: 160). Eine solche, die eigene Persönlichkeit weitgehend zurücknehmende Selbstinszenierung,

30 Das Bildungssystem vermittelt in erster Linie Allgemeinbildung. Die eigentliche fachliche Ausbildung erfolgt erst in den Betrieben. Dabei zielt diese nicht auf eine spezifische Berufsausbildung, sondern auf eine fortlaufende aufgabenbezogene „Anlernung" mit dem Ziel einer flexiblen Qualifikationsanpassung an und -verwertung durch die Organisation. Für die Mitglieder der Organisation heißt das, daß sie aus ihren vielfältigen, tendenziell unabschließbaren Bildungsbemühungen kein Kapital im Sinne von einklagbaren Statusansprüchen gegenüber dem Unternehmen schlagen können (Deutschmann 1987: 137).

31 Die Rekrutierung ,straight from school or college“ (Clark 1979: 156) bietet den Firmen erhebliche finanzielle Vorteile. Die Entlohnung bemißt sich nämlich u.a. nach dem Alter und der Dauer der Betriebszugehörigkeit. Insgesamt gesehen spielt das ökonomische Argument allerdings eine untergeordnete Rolle. zieht sich wie ein roter Faden durch das gesamte Bewerbungsverfahren.

So werden etwa die obligatorischen Auswahlaufsätze zum Thema „Meine Familie“ von den jungen Aspiranten genutzt, um sich selbst als relativ unreif und unerfahren darzustellen. Zwei Passagen mögen hier genügen: „I'm still a child beside my parents“, „I am a little too carefree, and I might be a little naive" (zit. nach Clark 1979: 161).

Solche Selbststilisierungen leiten stets zu dem vorweggenommenen moralischen commitment in das angestrebte Arbeitsverhältnis über. So lautet denn die Conclusio eines Bewerbungsaufsatzes: „As a product of the M-family (gemeint ist das Unternehmen, d.A.), I think that I can save my family but by going out into the world and working as hard as possible to contribute to a company and to society" (zit. nach Clark 1979: 161, eigene Hervorhebung).

Die hier referierten Beobachtungen lassen sich u.E. dahingehend interpretieren, daß die Firmen gegenüber Individualisierungsangeboten resistente Träger von Generalqualifikationen suchen. Die Bewerber hinwiederum versuchen, diesen Erwartungen zu entsprechen, indem sie sich als tendenziell biographielose Träger marktgängigen, rohen Qualifikations- und Subjektivitätsmaterials präsentieren.

$\mathrm{Zu}$ diesem Biographisierungsmodus der Biographielosigkeit führen zwei Wege:

Die erste Variante läuft darauf hinaus, die selbständige Steuerung der eigenen Biographie zurückzunehmen. So schildert Ishida den Fall einer Studentin, die in Bewerbungsgesprächen ihre vorgängigen Joberfahrungen nicht unerwähnt ließ, womit sie sich um Kopf und Kragen redete. Erst die Lektüre eines einschlägigen Beratungsbuches machte sie auf ihren Fehler aufmerksam. In den folgenden Bewerbungsgesprächen klammerte sie ihre Arbeitserfahrungen aus und gelangte so zum Ziel (Ishida 1986: 169).

Dahinter steht u.E. weitaus mehr als eine strategische Glättung des Lebenslaufs, die in jedem Bewerbungsgespräch opportun erscheint. Denn in dem Moment, in dem die Bewerberin ihre vorgängigen Erfahrungen leugnet, signalisiert sie gleichzeitig die Bereitschaft, subjektive Erfahrungen je nach Bedürfnissen der Organisation zu aktualisieren, zu ,vergessen“ oder auch kontingent zu halten. Außeruniversitär erworbene Qualifikationen sowie Berufserfahrungen werden gegenüber dem idealen Persönlichkeitsprofil des konturlosen und deshalb noch formbaren Bewerbers entwertet. 
Biographielosigkeit bedeutet also nicht, daß die Bewerber keine Lebensgeschichte haben dürfen. Es geht vielmehr darum, die eigene Lebensgeschichte so zu erzählen, daß man als „unbeschriebenes Blatt" erscheint.

Die zweite Variante stützt sich auf Sozialisationsprozesse, die sich im langen Schatten einer vorweggenommenen Berufskarriere entfalten und erst gar keinen Raum für eigenständige Erfahrungen lassen.

Ein so hochgradig hierarchisiertes, leistungsorientiertes Bildungssystem wie das japanische, das die Eintrittskarten für prestige- und einkommensträchtige Berufskarrieren verteilt, zwingt seine Schüler, von Kindesbeinen an enorme Bildungsanstrengungen auf sich zu nehmen. In vielen Familien sind solche Bemühungen in eine langfristige Bildungsplanung eingebettet. Laut Teichler wird „,von vielen Eltern ... bereits der Zugang zu einem bekannten Kindergarten als wichtiger Schritt angesehen“" (1986: 403).

Bewegen sich die Individuen aber immer nur in den engen Führungsschienen einer so einseitig zielgerichteten Sozialisationsgeschichte, wird es unwahrscheinlich, daß sie auf dem Weg zum erfolgreichen Abschluß ihrer Bildungsbemühungen solche Erfahrungen sammeln und Persönlichkeitsmerkmale ausbilden, die sich nicht bruchlos in den bereits erwähnten Erwartungshorizont des späteren Arbeitgebers einfügen. ${ }^{32}$

Beide Formen der früher oder später erworbenen Biographielosigkeit erlauben es den Unternehmen, während der langen betriebsinternen Ausbildungsphase nicht nur die fachliche Ausbildung, sondern auch die Umbildung der Persönlichkeitsstruktur ihrer Belegschaft in die Hand zu nehmen (vgl. Ishida 1986: 170f.). Das ,rohe Material" wird so lange geformt, bis es sich nahtlos in die Organisation einfügt. Im Zuge dieses Formungsprozesses geht die Differenz zwischen ,authentischem" Persönlichkeitskern und von der Organisation ange-

32 Dieses personalpolitische Leitbild wird anscheinend zunehmend mit Individualisierungs- und Besonderungstendenzen konfrontiert (Kreitz 1991). Christoph Deutschmann hat uns darauf hingewiesen, daß es den Unternehmen bis dato zu gelingen scheint, solche „Eigensinnigkeiten" durch das Ineinandergreifen der entgrenzten zeitlichen Beanspruchung durch den Clan, der damit einhergehenden sozialen Isolierung nach auBen und der Einbindung in das Regime des internen Gruppendrucks doch wieder einzuschmelzen. botenen Personalisierungsschablonen nahezu vollständig verloren. ${ }^{33}$

Neben der ,ausbaufähigen“ fachlichen Qualifikation (Stichwort ,Elitenrekrutierung“) und der psychischen Plastizität ist der biographische Hintergrund des Bewerbers ein weiteres entscheidendes Einstellungskriterium.

Mit dem peniblen Durchforsten der Bewerberbiographie (der Bogen läßt sich dabei von der besuchten Universität bis hin zum Reinlichkeitsverhalten der Eltern spannen, vgl. Deutschmann 1987: 136) verbindet sich vor allem die Absicht, solche Mitglieder zu rekrutieren, die die betriebsinterne Homogenität nicht stören. Denn die spontane Koordination der Clans setzt einen hohen Grad der Loyalität, vermittelt durch eine entsprechend intensiv gestaltete normative Integration der Mitglieder in das Organisationsgefüge, voraus. Unbedingte Loyalität und Fügsamkeit wächst nun aber am ehesten in einem homogenen Milieu, das - im Gegensatz zu einer heterogenen Personalstruktur und ihren vielfältigen Angeboten an konkurrierenden Bindungen - erst gar nicht zur Ausbildung privatistischer Besonderungswünsche oder gar individueller Ansprüche verführt.

Eine solche subjektive Disposition unterscheidet sich grundlegend von westlichen Modellen der Persönlichkeitsentwicklung. Deutschmann bezieht sich in diesem Zusammenhang auf Kohlbergs Überlegungen zur Ausbildung der postkonventionellen Moral, deren Entwicklungsmöglichkeit nur dort gegeben ist, , (...) wo das Individuum in der Lage ist, eine Vielzahl unterschiedlicher Erfahrungen zu machen und konkurrierende Loyalitäten einzugehen, die es dahin führen, sich von der Moral einer bestimmten Gruppe zu lösen und selbstreflexive Normen moralischen Verhaltens $\mathrm{zu}$ entwickeln“ (1989b: 390). In analoger Weise wird die reflexive Identität in funktional differenzierten Gesellschaften ausgebildet. Sie stellt sich folglich als „Exklusionsindividualität" dar (s. Abschnitt 1.).

Demgegenüber zeichnen sich Clans dadurch aus, $\mathrm{daB}$ sie private Motive schlucken, statt sie durch Disziplinierungen umzubiegen oder sie außen vor zu halten bzw. ins Informelle abzuspalten. Die hier diskutierte Form der Totalinklusion dehnt die Organisationsgrenze so weit aus, daß die Differenz zwischen Person und Organisation zu Lasten pri-

33 In ihrem Buch über große japanische Handelshäuser vermerken Yoshino und Lifson bezeichnenderweise, daß manche Japaner andere Zeitgenossen vom bloßen Augenschein mit erstaunlicher Treffsicherheit dem jeweiligen Arbeitgeber zuordnen können (1986: 142). 
vater Rückzugsmöglichkeiten eingeebnet wird. Der Clan verlangt explizit die ganze Person und bindet sie in starke, tendenziell den ganzen Lebenskreis umfassende biographische Skriptvorgaben ein. Aufgegeben wird das - laut Luhmann (1964) für funktional differenzierte Gesellschaften charakteristische - Mitgliedschaftsmodell der Inklusion qua Exklusion, (in dem, wie in den vorangegangenen Fallstudien gezeigt, die exkludierten Teile privater Lebensführung indirekt zu Ressourcen der Organisation werden) bzw. sein (lebensweltliches) Korrelat, das Modell der Exklusionsindividualität. An seine Stelle treten totalinklusive Mitgliedschaftsverhältnisse, die darauf beruhen, daß sich ein reflexiver Biographisierungsmodus mit dem Typus der organisationsvermittelten Inklusionsidentität verbindet. Darunter ist folgendes zu verstehen:

Die Totalinklusion begnügt sich nicht damit, daß Person und Organisation eins werden und die Person eine stabile, organisationsvermittelte soziale Identität annimmt. Das Besondere an diesem Inklusionsmodus ist vielmehr darin zu sehen, daß die persönliche Identität nahezu restlos in der organisationsvermittelten sozialen Identität eingeschmolzen wird. Dabei ist den Clans nicht daran gelegen, die Persönlichkeit ihrer Mitglieder in einer bestimmten (Berufs-)Rolle zu bannen bzw. sie ein für alle Mal darauf festzulegen. Ganz im Gegenteil: Die Persönlichkeit der Mitglieder und die in ihr gebundenen Ressourcen wie Kreativität, Spontaneität, Phantasie und vor allem die Fähigkeit zu reflexiver Selbststeuerung sollen erhalten und in der Organisation entfaltet werden.

Diese Begehrlichkeit zeigen neben den Clans natürlich auch andere Organisationen, doch ist das Spezifische der Clans darin zu sehen, daß die genannten Potentiale nicht in mehr oder weniger präzisen Mustern beruflicher Entwicklung (Karriere) eingehegt werden. Andere Organisationen müssen dazu Grenzen von Hierarchien, Exklusionsindividualitäten usw. überwinden, die in Clans erst gar nicht vorhanden sind. Der Clan nutzt die Persönlichkeitsressourcen seiner Mitglieder als Medium, das jederzeit in eine andere Form gebracht werden kann.

Dieser Organisationstypus zeichnet sich also dadurch aus, daß alle Elemente der Mitgliedschaft außer der Mitgliedschaft selbst, wie etwa Position, Aufgabenfeld, Weisungsbefugnisse, in jedem Moment verflüssigt und in einer veränderten Ausgestaltung wieder verfestigt werden können.

Ein solches Mitgliedschaftsverhältnis zielt auf ein dauerhaftes Präsenthalten interner Kontingenzen.
Oder um es in den Worten Deutschmanns zu sagen: Eine solche Organisation ist ,... nicht mehr nur an der Spitze, sondern auf jeder Entscheidungsebene in der Lage, die Luhmannschen Tugenden der Bürokratie auf sich selbst anzuwenden, also: Mit formalen Vorgaben reflektiv umzugehen, sie nicht als etwas, was so und nicht anders sein kann, sondern als eine unter anderen Möglichkeiten zu behandeln." Aufgabe der Mitglieder ist es, dies ,als einen selbstgesteuerten Prozeß zu entfalten“" (1987: 140).

Genau das ist der Grund, warum Clans biographielose Träger von Generalqualifikationen suchen. Sie stellen der Organisation ihre herausragenden kognitiven wie auch kreativen Fähigkeiten zur Verfügung. Gleichzeitig bringen sie einen Biographisierungsmodus mit, dessen Reflexivität sich darauf richtet, die eigene (Berufs-)Biographie als etwas kontingentes, jederzeit zur Disposition stehendes, veränderbares zu erleben und auch so $\mathrm{zu}$ behandeln. So gelingt es dem Clan, „Exzellenz“ (Peters, Waterman) ohne Rigidität dauerhaft $\mathrm{zu}$ binden.

Indem die „Schicksalsgemeinschaft“ (Deutschmann) Clan Sozialisation und Selektion in der skizzierten Weise verschränkt, gelingt ihr der Aufbau äußerst plastischer Mitgliedschaftsverhältnisse. Diese Plastizität erlaubt es, Mitgliedschaftsverhältnisse in ein frei verfügbares Humanpotential zu transformieren, das situativ variabel spezifiziert wird - und zwar, ohne daß von diesem Höchstmaß an interner Kontingenz eine Bedrohung für die fluide Stabilität der Organisation bzw. die Identität ihrer Mitglieder ausgeht. ${ }^{34}$

34 In der Darstellung des Clanmodells haben wir das Thema „Kultur“ weitgehend ausgespart. Damit soll natürlich nicht behauptet werden, daß der japanische Clan nicht auf bestimmten kulturellen Traditionsbeständen ruht, die ein günstiges Milieu für die Entfaltung totalinklusiver Mitgliedschaftsverhältnisse bilden. Ganz im Gegenteil: Solche kulturellen Muster wie etwa die starke Betonung der Gruppe, die den Wunsch nach individueller Besonderung in den Hintergrund treten läßt, die sehr moderate, diplomatisch-zurückhaltende Form der Konfliktaustragung (Kerbo/Wittenhagen/Nakao 1993: 33 f.) oder auch die spezifisch japanische Arbeitsorientierung, der bspw. eine strikte Trennung von Arbeits- und Freizeit fremd ist (Linhart 1989: 212), tragen und stützen entgrenzte Mitgliedschaftsverhältnisse. Dennoch möchten wir die kulturellen bzw. japanologischen Debatten hier nicht weiter verfolgen. In unserem Beitrag geht es ja nicht um eine Verhältnisbestimmung Kultur/Organisation, sondern, sofern das Thema Kultur überhaupt berührt ist, um die Frage, wie es einer Organisation gelingt, ein für ihre 
Übersicht: Dimensionen von Inklusionsverhältnissen.

\begin{tabular}{|c|c|c|c|c|}
\hline & Beamte & Fräulein vom Amt & Zeitarbeit & Betriebsclans \\
\hline Inklusion & total & partial & tangential & total \\
\hline $\begin{array}{l}\text { Rekrutierungs- } \\
\text { kriterien }\end{array}$ & $\begin{array}{l}\text { Herkunft; } \\
\text { Bildungszertifikate; } \\
\text { „Korrektheit“" }\end{array}$ & $\begin{array}{l}\text { Herkunft; } \\
\text { Mindestanforde- } \\
\text { rungen an Bildung } \\
\rightarrow \text { Belastbarkeit }\end{array}$ & $\begin{array}{l}\text { polyvalente } \\
\text { Qualifikationen; } \\
\text { Flexibilitätsbereit- } \\
\text { schaft }\end{array}$ & $\begin{array}{l}\text { Herkunft; } \\
\text { Bildungszertifikate; } \\
\text { psychische Plastizität }\end{array}$ \\
\hline $\begin{array}{l}\text { interne } \\
\text { Sozialisation }\end{array}$ & Vorbereitungsdienst & $\begin{array}{l}\text { Einarbeitung } \\
2-6 \text { Monate }\end{array}$ & - & internes Training \\
\hline $\begin{array}{l}\text { Dauer des Be- } \\
\text { schäftigungsver- } \\
\text { hältnisses }\end{array}$ & Lebenszeit & $\begin{array}{l}\text { Alternative Lebens- } \\
\text { zeit/Übergangsbe- } \\
\text { rufstätigkeit }\end{array}$ & $\begin{array}{l}\text { im Prinzip unbefristet; } \\
\text { faktisch begrenzt }\end{array}$ & Lebenszeit \\
\hline Karriere & $\begin{array}{l}\text { berechenbare } \\
\text { Laufbahn; } \\
\text { Senioritätsprinzip }\end{array}$ & - & $\begin{array}{l}\text { Interorganisations- } \\
\text { mobilität horizontal/ } \\
\text { vertikal }\end{array}$ & $\begin{array}{l}\text { diffuse Karrieremuster; } \\
\text { flache Hierarchien; } \\
\text { Senioritätsprinzip }\end{array}$ \\
\hline $\begin{array}{l}\text { Entlohnung und } \\
\text { soz. Absicherung }\end{array}$ & $\begin{array}{l}\text { Alimentierung; } \\
\text { Familienlohn }\end{array}$ & $\begin{array}{l}\text { diätarisch; } \\
\text { Alimentierung; } \\
\text { Individuallohn }\end{array}$ & $\begin{array}{l}\text { hohe Nettoeinkom- } \\
\text { men; soz. Absiche- } \\
\text { rung unterproportio- } \\
\text { nal dazu }\end{array}$ & $\begin{array}{l}\text { unternehmenserfolgs- } \\
\text { abhängige Gehaltsan- } \\
\text { teile; Familienlohn; } \\
\text { Renten nach Betriebs- } \\
\text { zugehörigkeit }\end{array}$ \\
\hline $\begin{array}{l}\text { charakteristischer } \\
\text { Tausch }\end{array}$ & $\begin{array}{l}\text { Fürsorge gegen } \\
\text { Treue }\end{array}$ & $\begin{array}{l}\text { Individualisierungs- } \\
\text { chancen gegen } \\
\text { Robustheit }\end{array}$ & $\begin{array}{l}\text { Toleranz gegen Per- } \\
\text { formanz/ } \\
\text { Freiheitskonzes- } \\
\text { sionen gegen Struk- } \\
\text { turierungspotential }\end{array}$ & $\begin{array}{l}\text { Geborgenheit gegen } \\
\text { Verfügbarkeit }\end{array}$ \\
\hline $\begin{array}{l}\text { symbolische } \\
\text { Ausstattung }\end{array}$ & $\begin{array}{l}\text { Profanethik und } \\
\text { Adelssymbole }\end{array}$ & evtl. "Modernität" & $\begin{array}{l}\text { teilweise: } \\
\text { „flexibler Lebensstil“ }\end{array}$ & $\begin{array}{l}\text { Familialisierung und } \\
\text { Moralisierung des Mit- } \\
\text { gliedschaftsverhältnis- } \\
\text { ses }\end{array}$ \\
\hline biogr. Skript & $\begin{array}{l}\text { institutionalisierte } \\
\text { Normalbiographie }\end{array}$ & $\begin{array}{l}\text { Übergangsberuf/ } \\
\text { alleinstehende } \\
\text { Berufsfrau }\end{array}$ & $\begin{array}{l}\text { vielfältig; Distanz zum } \\
\text { Normalarbeitsverhält- } \\
\text { nis }\end{array}$ & $\begin{array}{l}\text { „Schicksalsgemein- } \\
\text { schaft" }\end{array}$ \\
\hline $\begin{array}{l}\text { Wechselwirkung } \\
\text { Organisation und } \\
\text { Lebensführung }\end{array}$ & Wahlverwandtschaft & Chanceneröffnung & $\begin{array}{l}\text { Indifferenz' } \\
\text { Interpenetration }\end{array}$ & Symbiose \\
\hline
\end{tabular}

Belange günstiges kulturelles Milieu zu nutzen. Claudia Weber spricht in diesem Zusammenhang von einem ,selektiven Traditionalismus" in dem Sinne, ,... daß traditionelle Werte nicht quasi selbsttätig, ungebrochen wirksam sind, sondern ganz bewußt in den Dienst bestimmter Politiken gestellt werden" (Weber 1989: 222). Von Interesse sind folglich die institutionellen Vorkehrungen, die traditionellen kulturellen Werten eine moderne Durchschlagkraft verleihen, wobei eine dieser institutionellen Vorkehrungen eben das Ineinandergreifen von Selektion und Sozialisation mit dem Ziel einer "Rundumnutzung“ der Arbeitskraft (Weber 1989) ist.

\section{Synopse und Schluß}

In der Übersicht haben wir die von uns behandelten Dimensionen und Aspekte der verschiedenen Inklusionsverhältnisse noch einmal zusammengestellt.

In zwei Fällen - den Beamten und dem Betriebsclan - finden wir den Modus der "Totalinklusion“. Personen werden in ihrer Gesamtheit von der Organisation in Anspruch genommen, und ihre Lebensführung bzw. Biographie entwickelt sich entweder ko-evolutionär zur Organisation oder 'aber in deren Schatten. In den beiden anderen Fällen, 
dem der „Fräulein vom Amt" und der „Zeitarbeit" sprechen wir von einer partialen bzw. tangentialen Inklusion. Personen werden hier selektiv, also nur unter Einbeziehung von Teilaspekten ihrer gesamten Lebensführung an die Organisation angeschlossen bzw. geraten - wie im Falle der Zeitarbeii - nur kurzfristig und punktuell mit der Organisation in Berührung.

Was die sachlichen, zeitlichen und sozialen Dimensionen des Mitgliedschaftsverhältnisses anbelangt, so läßt sich anhand der Rekrutierungskriterien und der Intensität bzw. Dauer der innerhalb der Organisation sich anschließenden Sozialisationsprozesse ablesen, wie selektiv die Filter sind, die die Organisation benutzt, wenn sie Personen zu Mitgliedern macht. Auch hier ergibt sich, was nicht überrascht, eine hohe Ähnlichkeit zwischen den beiden Formen der "totalen“ Inklusion. Die entscheidende Differenz scheint jedoch in der Frage zu liegen, inwiefern bestimmte Formen persönlicher und organisationeller „Rigidität“ von der Organisation als funktional betrachtet werden. Während in den japanischen Betriebsclans die psychische und soziale Plastizität genutzt wird, gilt für die Beamten eine Verhaltenserwartung, die man als habitualisierte „Korrektheit“ bezeichnen kann.

Auch die klare Abgrenzung der ihnen jeweils zugewiesenen - grundsätzlich aber durchaus verschiedenen - Aufgaben verweist auf die festen Regeln, die vorschreiben, was in der Organisation "Sache" ist, und wie damit zu verfahren sei. Für die „Fräuleins vom Amt“ gilt dies in einem spezifischen Sinn. Hier geht es nicht um die ,routinierte Festigkeit" im Umgang mit jeweils zugewiesenen Aufgaben, sondern um Spezialisierung in der Ausübung der gleichen Funktion. Die Rekrutierung erfolgt denn auch - nach anfänglicher Orientierung an der kleinbürgerlichen Herkunft der Bewerberinnen - nach dem Kriterium der psychophysischen Robustheit. Gerade weil hier die Inklusion von Personen selektiv erfolgt, ist eine Spezialisierung in der Aufgabenerfüllung denkbar bzw. umgekehrt: Weil Spezialisierung gefordert ist, werden nur bestimmte Formen der Lebensführung zugelassen. In der Zeitarbeit dagegen kann die Aufgabenstruktur hochgradig variieren. Es muß immer gerade die Arbeit erfüllt werden, für die es in der Nutzerorganisation Bedarf gibt, der durch extern abrufbare Leistungen befriedigt werden kann. In den Betriebsclans ist die Aufgabenprofilierung auch für den dauerhaften Stelleninhaber unscharf. Die variierende Struktur der Aufgabenerfüllung soll mit internen Mitteln bearbeitet werden. Deshalb soll die vielseitige Einsetzbarkeit der Mitglie- der auf Dauer gewährleistet werden. Spezialisierungen und Rigiditäten wären da nur hinderlich. In dieser unterschiedlichen Schärfe der Abgrenzung von Aufgaben, also der Definition dessen, was Sache ist und was nicht, ergeben sich verschiedene Möglichkeiten, Tätigkeiten innerhalb der Organisation mit Sinn anzureichern, v.a. auch mit Sinn, der auf Lebenszusammenhänge jenseits der Organisation verweist.

Diese Unterschiedlichkeit in der Verweisung auf andere Kontexte kommt auch - in der sozialen Dimension - bei den verschieden profilierten Anreizsystemen zum Ausdruck. Bei der Strukturierung und Dimensionierung von Erwartungen, der Stimulierung von Kooperations- und Leistungsbereitschaft wie auch in der spezifischen Gestaltung des sozialen Tausches, sind die Berücksichtigung von Aspekten jenseits von Lohn und Leistung und die Einbeziehung unterschiedlicher Ausschnitte der ,außerbetrieblichen“ Lebenswelt bedeutungsvoll. Dies gilt - wenn man auch die zeitliche Dimension des Mitgliedschaftsverhältnisses berücksichtigt - verstärkt für die langfristig wirksamen Anreizmechanismen, etwa Senioritätsregelungen oder Beschäftigungsgarantien. Sie entfalten Bindungswirkungen und eröffnen relativ sichere Erwartungshorizonte. Entwürfe, Erwartungen und Erfahrungen im lebenszeitlichen Horizont der Personen rechnen dann auch immer mit Ressourcen, Sicherheiten und Beschränkungen, die durch die Bindung an eine Organisation wirksam werden. Und die Organisation, die durch ihre Festlegung auf bestimmte Personen Liquiditätsverzichte erbringt, kann auf diese Weise ihre Fluktuationskosten kontrollieren und ansonsten schwer mobilisierbare Beitragsmotivationen erschließen. Hier sind also Wechselwirkungen zwischen Organisation und Biographie besonders wahrscheinlich und auch deutlich erkennbar.

In der Form des Beamtentums finden diese statt, indem die sich entwickelnde, bürokratische Organisation auf Muster der Lebensführung zurückgreift, die sich in spezifischen sozialen Milieus entwickelt haben und durch sozialisatorische Prozesse in solchen, autochthonen Sozialmilieus geprägt sind. Hier ergibt sich das, was man in Anlehnung an Max Weber als eine Wahlverwandtschaft von Lebensführung und Organisationstypus bezeichnen kann. Während wir im Fall des männlichen Beamten zunächst eine spannungsreiche Komplementarität von Organisation und Lebensführung fanden, die später zu einer Identität von Organisations- und Persönlichkeitsstruktur verflacht (bürokratischer Habitus), sehen wir im weiblichen Mo- 
dell der auf Ehelosigkeit verpflichteten Beamtin eine definitorische Vorgabe der Lebensführung durch die Organisation. Hier impliziert die Inklusion die Exklusion. Davon abzweigend entwickelt sich das „Normal"-Modell der weiblichen Übergangsbeschäftigung, das Ausdruck empirischer, kontingenter Organisationsentwicklung ist. Unser Beispiel der Zeitarbeit sollte dann zeigen, daß hier - man könnte sagen: eigentlich erst hier - von einer nahezu reinen vertragsförmigen Beziehung gesprochen werden kann. ${ }^{35}$

Die Lebensführung der Mitglieder und die Organisation können hier - zumindest im Arbeitsverhältnis - nebeneinander koexistieren oder gar so weitgehend divergieren, wie es irgend möglich scheint ${ }^{36}$ Eine Ko-Evolution von Lebensstil und Organisation ist - so nehmen wir an - aber für bestimmte Konstellationen und Kombinationen denkbar. Da nämlich, wo Personen, die in ihrer Biographie die Bewältigung von Diskontinuitäten und den Umgang mit fragmentierten Lebenswelten gelernt haben, diese Strukturierungsfähigkeiten der Organisation gewissermaßen zur Verfügung stellen. Dies könnte man dann, im spezifischen Sinne des Begriffs, Interpenetration nennen: Persönlichkeitssystem und Organisation stellen sich wechselseitig Komplexität zu Verfügung, nutzen einander jeweils für ihren Strukturaufbau, ohne daß Systemgrenzen verändert, neue Systeme gebildet würden. Demgegenüber scheint die Entwicklungsvariante der Clans eine Form totaler Einbindung darzustellen. Sie erinnert an jene auf der Identität von Person und Organisation basierende Inklusion, wie wir sie an den Entwicklungsvarianten des Beamtenmodells dargestellt haben, bei denen soziale und persönliche Identität verschmelzen (der weiblichen mit Eheverbot und der männlichen in ihrer Version des bürokratischen Habitus). Dennoch gibt es eine wichtige Differenz. Die bürokratische Persönlichkeitsstruktur ist fi-

35 Vorsichtshalber sei noch einmal (vgl.o. 1.) daran erinnert, daß sich bei den meisten Mitgliedschaftsverhältnissen in formalen Arbeitsorganisationen aufgrund der Besonderheiten des Arbeitsvertrages, nämlich seiner Unbestimmtheit, eine Palette von Mitgliedschaftsrollen ausdifferenziert, die jeweils weit über das reine Vertragsmodell hinausgehen, Elemente statusförmiger Integration enthalten und damit die biographische Entwicklung der Beschäftigten beeinflussen können.

36 Eine Einschränkung muß hier insofern gemacht werden, als durch das „beschäftigende“ Verleihunternehmen natürlich eine Bewerberauswahl stattfindet, die aber gerade im Hinblick auf biographische Aspekte nicht selektiv wirkt (vgl. Brose et al. 1993). xiert in ihren Verfahrensregeln. Der Bürokrat kann nur bürokratisch sein. Die clanförmige Totalinklusion japanischer Prägung scheint aber in ihrer Bindung anders zu funktionieren. Die Bindung ist pauschaler, wenn man will: abstrakter. Definiert wird nicht, was jemand tut und wie, sondern daß, was immer er oder sie tut, es als Mitglied der Organisation getan wird. Es werden nicht Individuen sozialisiert und eingepaßt, sondern Humanpotential wird organisations- und situationsadäquat jeweils spezifiziert: totale Bindung und hohe Plastizität. Im Modell des Betriebsclans könnte man das Verhältnis von Personen und Organisation als Symbiose (Katz/Kahn 1966) kennzeichen, als eine „organische" Einheit, in der die Entwicklungen von Personen und Organisationen schicksalhaft verknüpft sind.

Unser Anliegen war analytischer, nicht prognostischer Art. Anhand der von uns skizzierten Entwicklungsvarianten der Inklusionsverhältnisse von Biographien und Organisationen lassen sich keine Aussagen über die Richtung dieser Entwicklung ableiten. Die von uns angeführten Beispiele der Zeitarbeit einerseits und der Clans andererseits können aber als Pole eines Kontinuums angesehen werden, zwischen denen sich andere Entwicklungsvarianten einordnen lassen. Durch diese Veränderung des Blickwinkels erscheint dann das, was in der herrschenden Diskussion, mit Bezug auf das Modell der formalen Organisation, häufig nur als empirische Abweichung gesehen wird, als eine von vielen möglichen Entwicklungsvarianten. Und die Variante, die dem Modell am ehesten entspricht, die Zeitarbeit, erscheint uns als eine unwahrscheinliche und hochspezifische Konstruktion.

\section{Literatur}

Adorno, Th. W., 1972 [1953]: Individuum und Organisation. S. $441-456$ in Gesammelte Schriften, Band 8, Frankfurt/M.: Suhrkamp

Antrag 1895: Allerunterthänigster Antrag des Staatsministeriums des Königlichen Hauses und des Aeussern Die Beschäftigung weiblicher Kräfte im TelephonUmschaltedienste betreffend an Seine Kgl. Hoheit den Prinzen Luitpold vom 11.Juni 1895, Akten der OPD München, Bayerisches Staatsarchiv

Argyris, Ch., 1957: Personality and Organization - The Conflict Between System and the Individual, New York: Harper

Argyris, Ch., 1973: Personality and Organization Theory Revisited. Administrative Science Quarterly 18: 141-67

Balazs, G./Faguer, J.-P., 1991: Des Enterprises sans Paternalisme? La Gestion de la vie Familiale Par les Enter- 
prises Meritocratiques. Centre d'Etudes d'Emploi: Dossier De Recherche, no 40, Nov.

Bleek, W., 1972: Von der Kameralausbildung zum Juristenprivileg. Studium, Prüfung und Ausbildung der höheren Beamten des allgemeinen Verwaltungsdienstes in Deutschland im 18. und 19. Jahrhundert, Berlin: Colloquium

Braun, F., 1912: Die Frau im Staatsdienst, dargestellt an den Verhältnissen bei der Reichs-Post- und Telegraphenverwaltung, Berlin (Diss.)

Brose, H.-G./Schulze-Böing, M./Meyer, W., 1990: Arbeit auf Zeit, Opladen: Leske \& Budrich

Brose, H.-G./Wohlrab-Sahr, M./Corsten, M., 1993: Soziale Zeit und Biographie, Opladen: Westdeutscher Verlag

Clark, R., 1979: The Japanese Company, New Haven, London: Yale University Press

Deutschmann, Ch., 1987: Der „Betriebsclan“. Der japanische Organisationstypus als Herausforderung an die soziologische Modernisierungstheorie. Soziale Welt 38: 133-147

Deutschmann, Ch., 1989a: Der „Clan“ als Unternehmens modell der Zukunft? Leviathan 17: 85-107

Deutschmann, Ch., 1989b: Reflexive Verwissenschaftlichung und kultureller Imperialismus des Managements. Soziale Welt 40: 375- 396

Dragendorf, R./Heering, W., 1987: Beschäftigungsdauer, Effizienz und Flexibilität. S.121-156 in Buttler, F. (Hrsg.): Arbeitsmarkt und Beschäftigung, Frankfurt/ M.- New York: Campus

Durkheim, E., 1977: Über die Teilung der sozialen Arbeit, Frankfurt/M.: Suhrkamp

Gabriel, K., 1979: Analysen der Organisationsgesellschaft: Ein kritischer Vergleich der Gesellschaftstheorien Max Webers, Niklas Luhmanns und der phänomenologischen Soziologie, Frankfurt/M. - New York: Campus

Gold, H., 1993: „Fräulein vom Amt" - eine Einführung zum Thema. S. 10-36 in H. Gold/A. Koch (Hrsg.), Fräulein vom Amt, München: Prestel

Gold, H./Koch, A. (Hrsg.), 1993: Fräulein vom Amt, München: Prestel

Hattenhauer, H., 1980: Geschichte des Beamtentums, Köln u.a.: Heymanns

Hausen, K., 1980: Die Polarisierung der „Geschlechtscharaktere". Eine Spiegelung der Dissoziation von Familien- und Erwerbsleben. S. 161-191 in Rosenbaum, H. (Hrsg.), Seminar Familie und Gesellschaftsstruktur. Materialien zu den sozioökonomischen Bedingungen von Familienformen, Frankfurt: Suhrkamp

Hohn, H.-W./Windolf, P., 1988: Lebensstile als Selektionskriterien - Zur Funktion biographischer Signale in der Rekrutierungspolitik von Arbeitsorganisationen. S. 179-210 in H.-G. Brose/B. Hildenbrand (Hrsg.): Vom Ende des Individuums zur Individualität ohne Ende, Opladen: Leske \& Budrich

Holtgrewe, U., 1989: Die Arbeit der Vermittlung. Frauen am Klappenschrank. Hessische Blätter für Volks- und Kulturforschung Bd.24: 113-124

Ishida, H., 1986: Educational Credentials, Class, and the Labor Market: A Comparative Study of Social Mobili- ty in Japan and the United States, Cambridge Mass.: Diss. Harvard University

Katz, D./Kahn, R. L., 1966: The Social Psychology of Organizations, New York u.a.: John Wiley

Kerbo, H. R./Wittenhagen, E./Nakao, K., 1993: Japanese Corporations in Germany: Corporate Structure and Employee Relations, Duisburg: Duisburger Beiträge zur soziologischen Forschung, No.7/1993

Kieser, A., 1989: Organizational, Institutional, and Societal Evolution. Administrative Science Quarterly 34: 540-564

Knoke, D./Prensky, D.; 1984: What Relevance do Organization Theories Have for Voluntary Associations? Social Science Quarterly 65: 3-20

Koch, A., 1993: „Arbeiten und Frohsein“ - Der Verband der deutschen Reichs-Post- und Telegraphenbeamtinnen. S. 56-66 in H. Gold/A. Koch (Hrsg.), Fräulein vom Amt, München: Prestel

Kocka, J., 1969: Unternehmensverwaltung und Angestelltenschaft am Beispiel von Siemens 1847-1914, Stuttgart: Klett-Cotta

Kreitz, S., 1991: Adoleszenzkultur in Japan. S.69-87 in Veröffentlichungen des Japanisch-Deutschen Zentrums Berlin, Band 14 (Wissenschaftliche Jahrestagung "Individualisierung in der japanischen Gesellschaft", 4.-6.12.1991), Berlin

Linhart, S. 1989: Die Anwendbarkeit des Freizeitbegriffs auf Japan. Leviathan 17: 204-215

Luhmann, N., 1964: Funktionen und Folgen formaler Organisation, Berlin: Duncker \& Humblot

Luhmann, N., 1973 [1968]: Zweckbegriff und Systemrationalität: Über die Funktion von Zwecken in sozialen Systemen, Frankfurt: Suhrkamp

Luhmann, N., 1981: Organisation und Entscheidung. S. 335-389 in ders., Soziologische Aufklärung 3. Soziales System, Gesellschaft, Organisation, Opladen: Westdeutscher Verlag

Luhmann, N., 1989: Individuum, Individualität, Individualismus. in ders.: Gesellschaftsstruktur und Semantik, Band 3, Frankfurt/M.: Suhrkamp

Luhmann, N., 1989: Gesellschaftsstruktur und Semantik. Studien zur Wissenssoziologie der modernen Gesellschaft, Band 3. Frankfurt/M.: Suhrkamp

Mayntz, R., 1985: Soziologie der öffentlichen Verwaltung, Heidelberg: Müller

Milles, D., 1993: Nervenbelastung oder nervöse Veranlagung - das medizinische Urteil über Telefonunfälle und Telefonistinnenkrankheiten. S.94-108 in $\mathbf{H}$. Gold/A. Koch (Hrsg.), Fräulein vom Amt, München: Prestel

Mintzberg, H.M., 1983: The Structure in Fives: Designing Effective Organizations, Englewood Cliffs N.J.: Prentice-Hall

Nienhaus, U., 1990: „Unter dem Reichsadler“. Postbeamtinnen und ihre Organisation 1908-1933. 1999. Zeitschrift für Sozialgeschichte des 20. und 21. Jahrhunderts 3/1990: 56-79

Nienhaus, U., 1993a: Das „Fräulein vom Amt“ im internationalen Vergleich, S.37-55 in H. Gold/A. Koch (Hrsg.), Fräulein vom Amt, München: Prestel 
Nienhaus, U., 1993b: Vater Staat und „KAPOVAZ““: Die Post als Vorreiter seit der Jahrhundertwende. S.69-84 in K. Hausen/G. Krell (Hrsg.), Frauenerwerbsarbeit. Forschungen zu Geschichte und Gegenwart, München: Hampp

Ouchi, W.G., 1980: Markets, Bureaucracies, and Clans. Administrative Science Quarterly 25: 129-141

Ouchi, W.G., 1981: Theory Z. How American Business Can Meet The Japanese Challenge, Reading Mass. u.a.: Addison-Wesley

Ouchi, W.G./Jaeger, A.M., 1978: Type Z Organization: Stability in the Midst of Mobility. Academy of Management Review 3: 305-314

Perrow, Ch., 1979: Complex Organizations. A Critical Essay, Glenview, Illinois: Scott, Foresman \& Company

Perrow, Ch., 1991: A Society of Organizations. Theory and Society 15: 725-762

Rosenbaum, H., 1982: Formen der Familie. Untersuchungen zum Zusammenhang von Familienverhältnissen, Sozialstruktur und sozialem Wandel in der deutschen Gesellschaft des 19.Jahrhunderts, Frankfurt:Suhrkamp

Sachse, C., 1988: A Flow of People and a Flow of Goods. Factory Family Policy at Siemens, 1918-1945. S. 65-81 in T. Siegel (Hrsg.), Fordism and Fascism, Armonk N.Y.: M. E. Sharpe

Schimank, U., 1981: Identitätsbehauptung in Arbeitsorganisationen - Individualität in der Formalstruktur, Frankfurt/M. - New York: Campus

Siegel, T., 1993: Das ist nur rational. Ein Essay zur Logik der sozialen Rationalisierung. S.363-396 in D. Reese u.a. (Hrsg.): Rationale Beziehungen? Geschlechterverhältnisse im Rationalisierungsprozeß, Frankfurt/ M.: Suhrkamp

Stichweh, R., 1988: Inklusion in Funktionssysteme der modernen Gesellschaft. S. 261-293 in R. Mayntz u.a.: Differenzierung und Verselbständigung, Frankfurt/M., New York: Campus

Streeck, W., 1988: Status und Vertrag als Grundkategorien einer soziologischen Theorie der industriellen Beziehungen, discussion paper, WZB Berlin, FS I 88-3, Berlin
Teichler, U., 1986: Das Bildungswesen. S.397-406 in M. Pohl (Hrsg.): Japan. Geographie - Geschichte - Kultur - Religion - Staat - Gesellschaft - Bildungswesen - Politik - Wirtschaft, Stuttgart, Wien: Thienemann

Twellmann, M., 1993 [1972]: Die deutsche Frauenbewegung. Ihre Anfänge und erste Entwicklung 1843-1889, Frankfurt/M.: Hain

Voß, G.-G., 1991: Lebensführung als Arbeit. Über die Autonomie der Person im Alttag der Gesellschaft, Stuttgart: Enke

Wagner, O., 1913: Die Frau im Dienste der Reichs-Postund Telegraphenverwaltung, Halle (Diss.)

Weber, C., 1989: Arbeit und Freizeit in Japan. Leviathan 17: $216-225$

Weber, M., 1988 [1904/1924]: Agrarstatistische und sozialpolitische Betrachtungen zur Fideikommißfrage in Preußen, in M. Weber 1924: Gesammelte Aufsätze zur Soziologie und Sozialpolitik, photomech. Nachdruck 1988, Tübingen: Mohr

Weber, M., 1972: Wirtschaft und Gesellschaft, Tübingen: Mohr

Weisungen 1885-1917: Weisungen für Umschalte- und Fernleitungsstelle 1885-1917, Nürnberg: Postmuseum

Westphalen, R. Graf von, 1979: Akademisches Privileg und demokratischer Staat. Ein Beitrag zur Geschichte und bildungspolitischen Problematik des Laufbahnwesens in Deutschland, Stuttgart: Klett-Cotta

Wunder, B., 1974: Die Entstehung des modernen Staates und des Berufsbeamtentums in Deutschland im frühen 19. Jahrhundert. Leviathan 2: 459-478

Wunder, B., 1977: Die Rekrutierung der Beamtenschaft in Deutschland. Leviathan 5: 360-377

Wunder, B., 1986: Geschichte der Bürokratie in Deutschland, Frankfurt: Suhrkamp

Yoshime, K./Schönbauer, G., 1994: Lebensstilwandel im modernen Japan. Anmerkungen zu Bourdieu aus japanischem Blickwinkel. Oita University Economic Review 1994: 49-81

Yoshino, M.Y./Lifson, Th.B., 1986: The Invisible Link. Japan's Sogo Shosha and the Organization of Trade, Cambridge, Mass., London: MIT University Press 